\title{
Comparing the Intermediate Precision in Petroleomics by Ultrahigh Resolution Mass Spectrometry
}

Gabriely S. Follia,b, Lindamara M. Souza ${ }^{a, b}$, Bruno Q. Araújoa, Boniek G. Vaz ${ }^{c}$, Paulo R. Filgueiras, ${ }^{a}$ and Wanderson Romão*a,b,d

aLABPETRO - Laboratory of Research and Development of Methodologies for Petroleum Analyses, Chemistry Department, Federal University of Espírito Santo, Av. Fernando Ferrari, 514, Goiabeiras, Vitória, ES - Brazil; P.O. Box: 29075-910.

bLaboratório de Petroleômica e Química Forense, Departamento de Química, Universidade Federal do Espírito Santo, 29075-910, Vitória, ES, Brazil.

IInstituto de Química, Universidade Federal de Goiás, 74001-970 Goiânia-GO, Brazil.

dInstituto Federal do Espírito Santo (IFES), Av. Ministro Salgado Filho, Soteco, Vila Velha, ES 29106-010, Brazil.

*E-mail: wandersonromao@gmail.com

\section{SUPPORT INFORMATION}

\section{Table of Contents}

Figures S1-14

Table S1-S10 


\section{Supporting Figures and Tables}
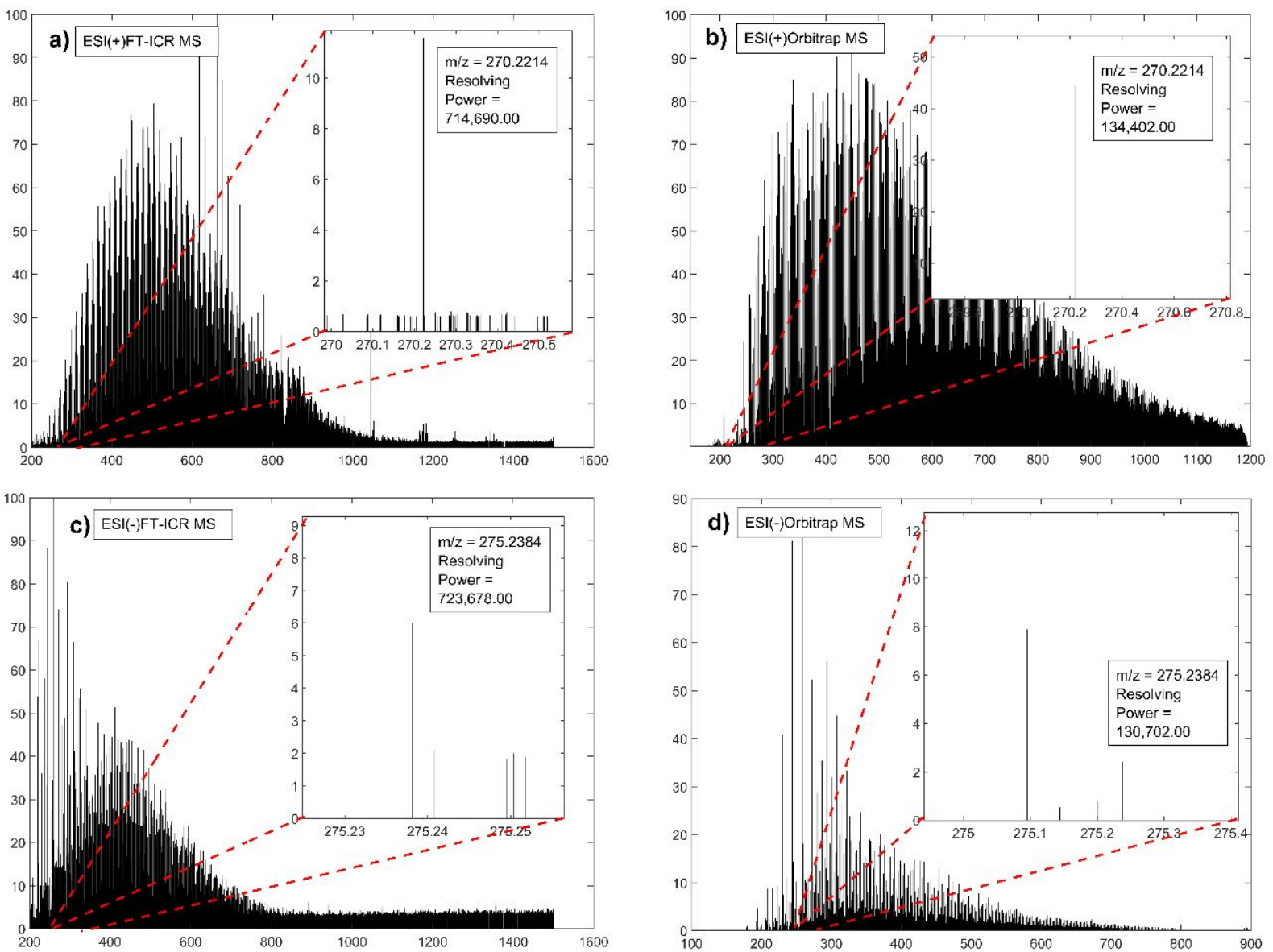

Figure S1. Resolving power of the same $\mathrm{m} / \mathrm{z}$ for both $\mathrm{ESI}( \pm)$-coupled mass spectrometers. 

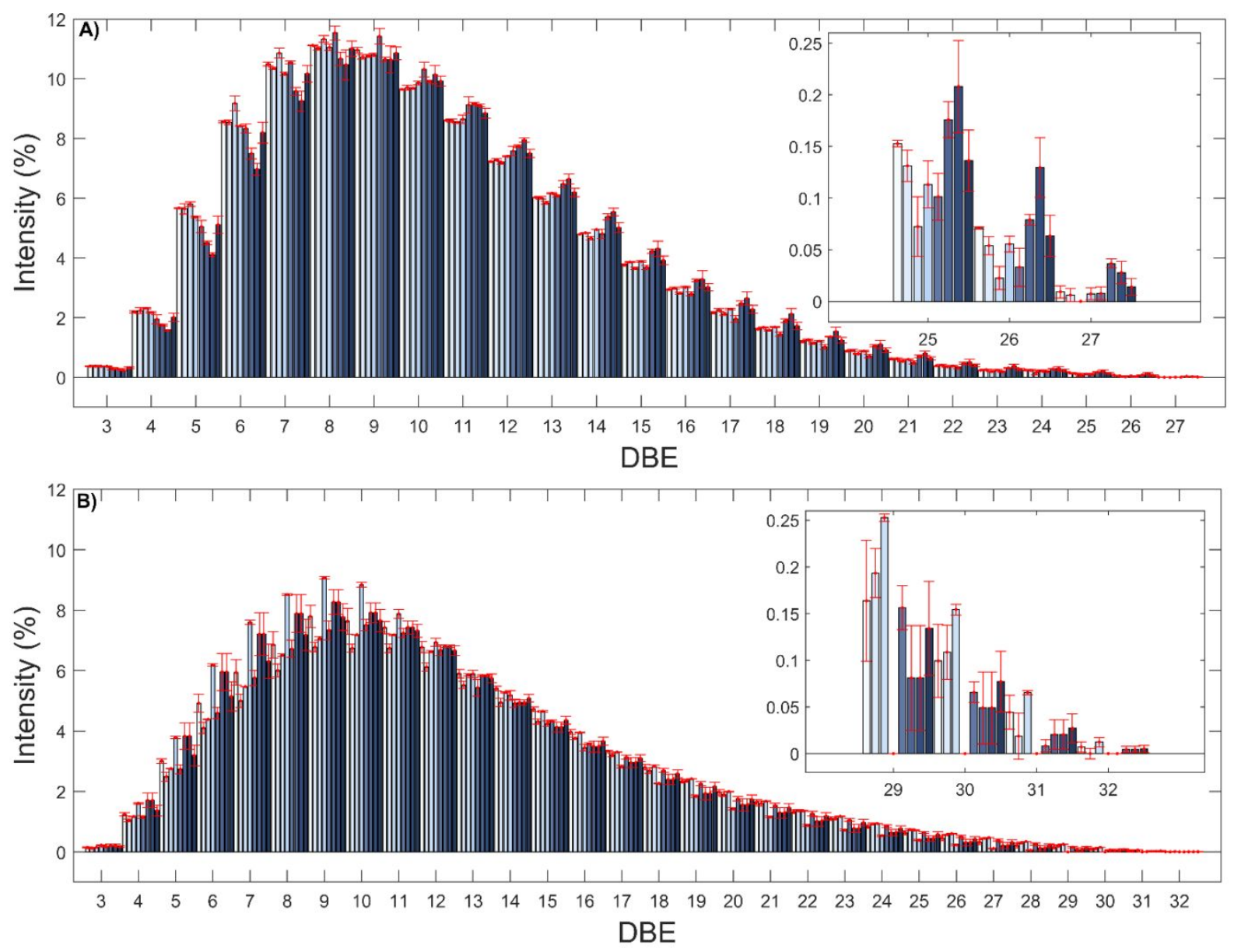

Figure S2. Plot of DBE distribution intensities of the majority class $\mathrm{N}[\mathrm{H}]$ by $(\mathrm{A}) \mathrm{ESI}(+) \mathrm{FT}-\mathrm{ICR}$ MS and (B) ESI(+)Orbitrap MS for the averages $(n=3)$ for each day of analysis for Crude oil A. 

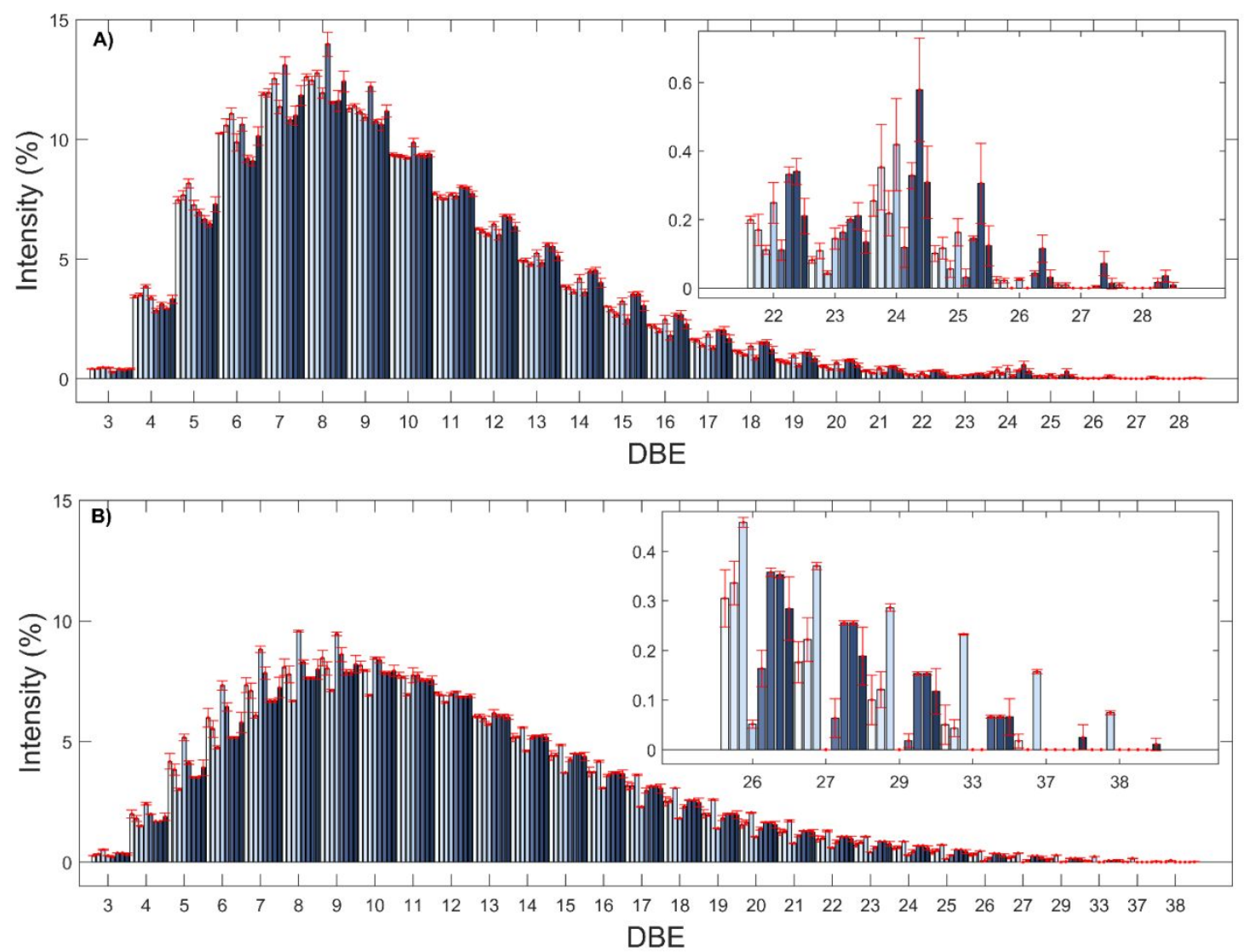

Figure S3. Plot of DBE distribution intensities of the majority class $\mathrm{N}[\mathrm{H}]$ by $(\mathrm{A}) \mathrm{ESI}(+) \mathrm{FT}$ ICR MS and (B) ESI(+)Orbitrap MS for the averages $(n=3)$ for each day of analysis for Crude oil B. 

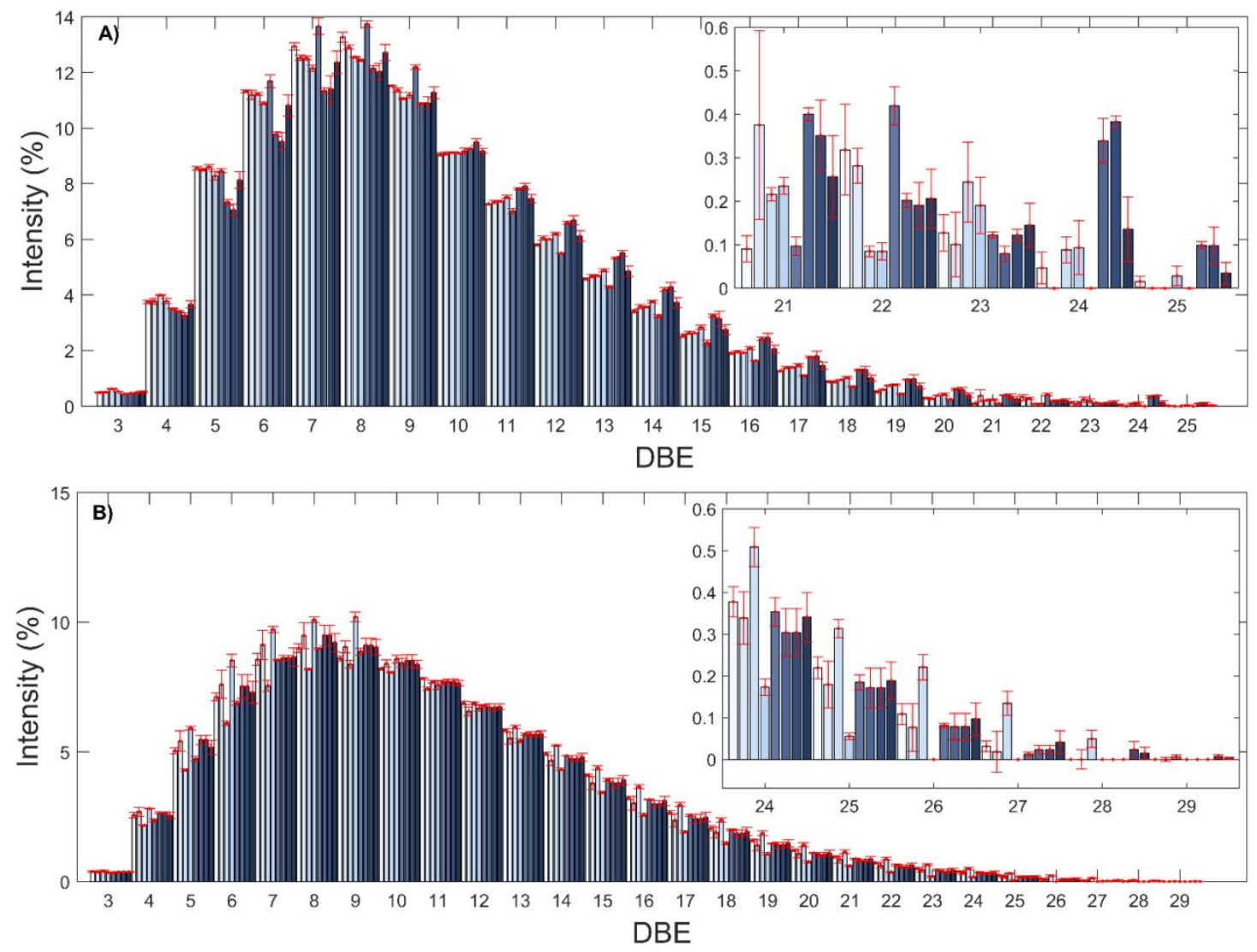

Figure S4. Plot of DBE distribution intensities of the majority class $\mathrm{N}[\mathrm{H}]$ by $(\mathrm{A}) \mathrm{ESI}(+) \mathrm{FT}-$ ICR MS and (B) ESI(+)Orbitrap MS for the averages $(n=3)$ for each day of analysis for Crude oil C. 

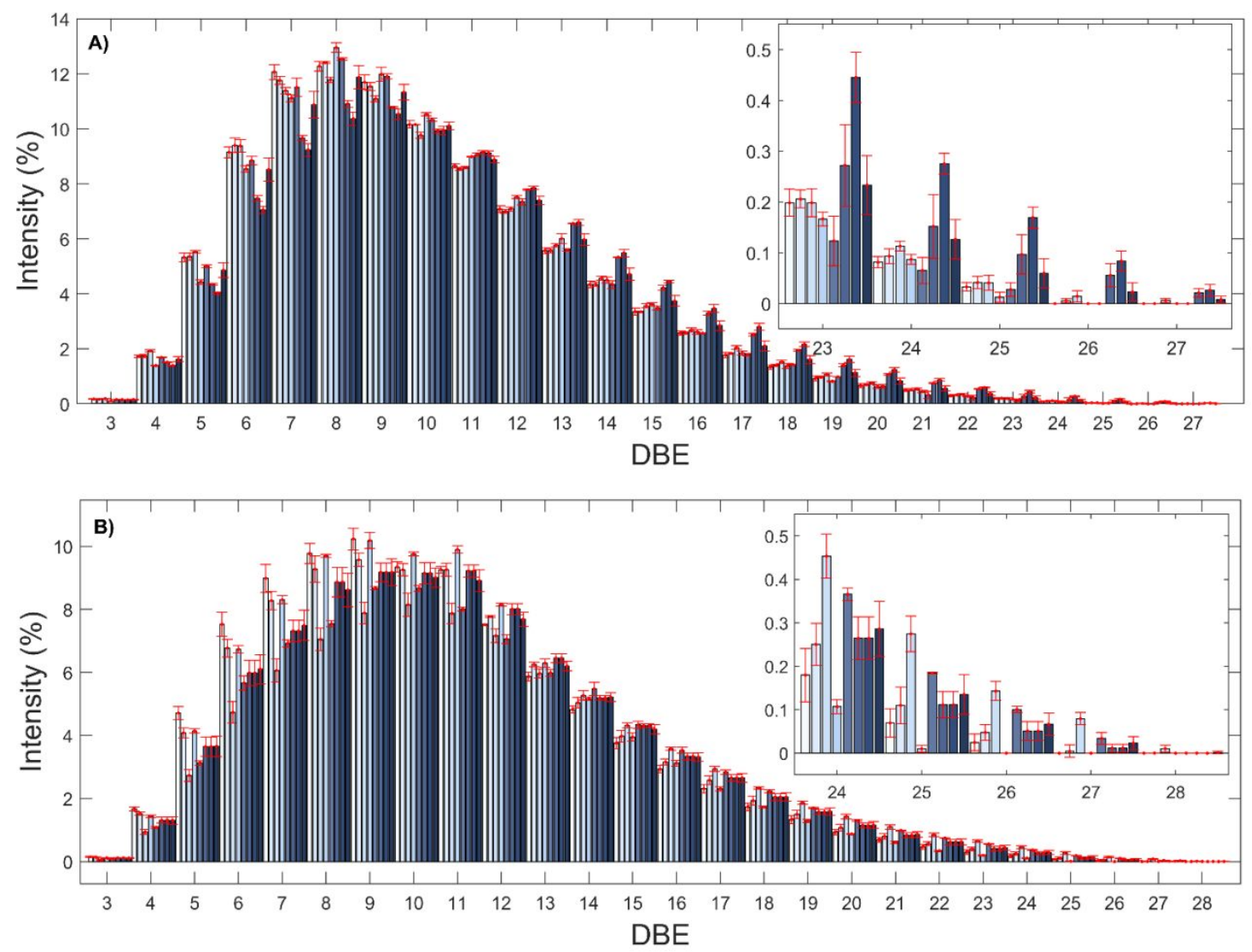

Figure S5. Plot of DBE distribution intensities of the majority class $\mathrm{N}[\mathrm{H}]$ by $(\mathrm{A}) \mathrm{ESI}(+) \mathrm{FT}-$ ICR MS and (B) ESI(+)Orbitrap MS for the averages $(n=3)$ for each day of analysis for Crude oil D. 


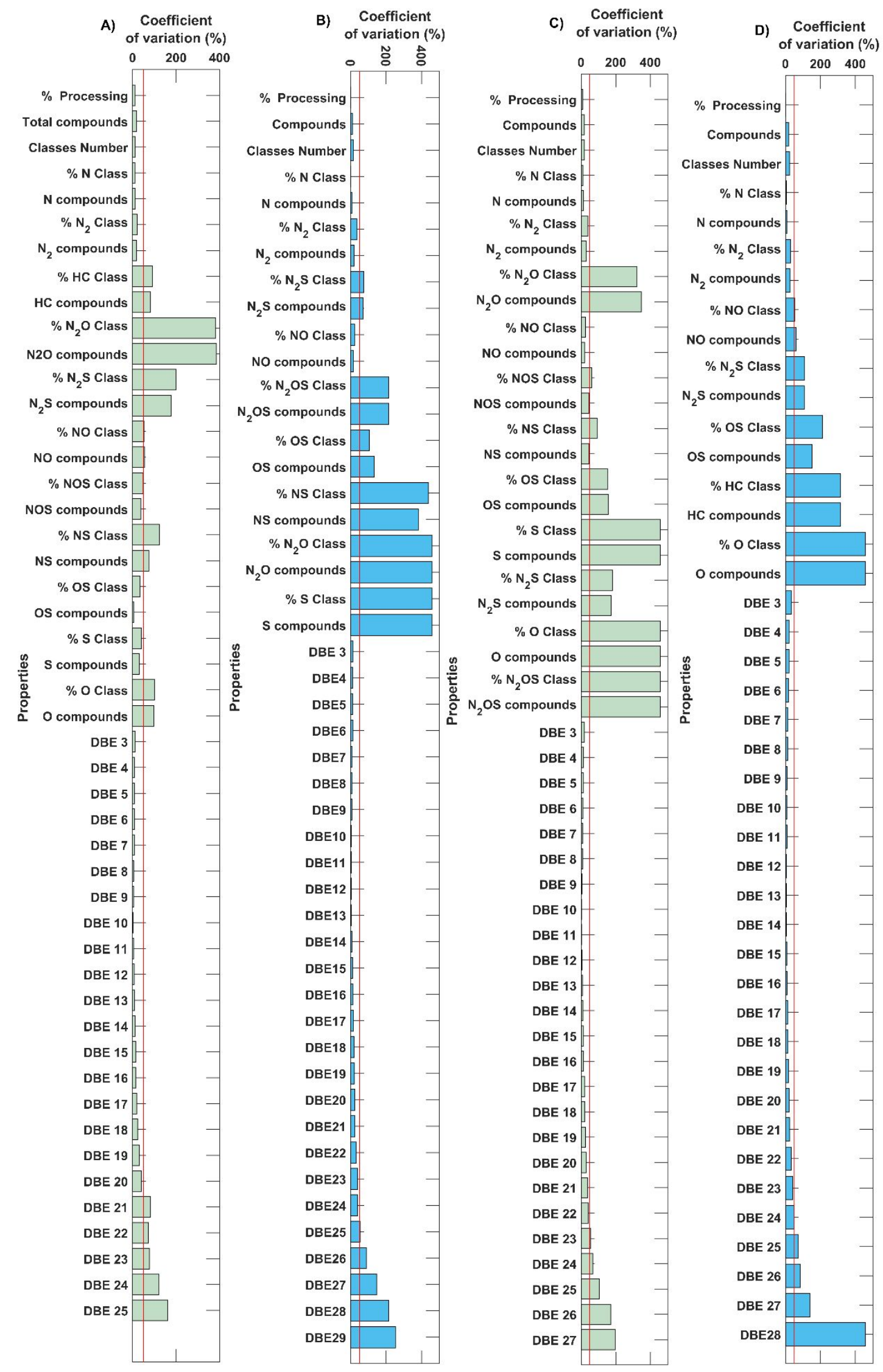

Figure S6. Plot of CV values of 21 analyzes by $(A, C)$ ESI(+)FT-ICR MS (Green bar) MS and $(B, D) E S I(+)$ Orbitrap MS (Blue bar) for Crude oil $C(A, B)$ and $D(C, D)$. 
A)

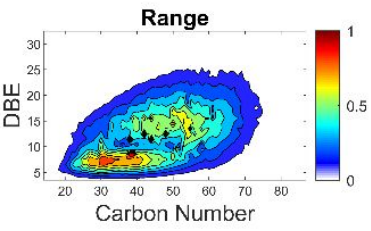

B) 岗
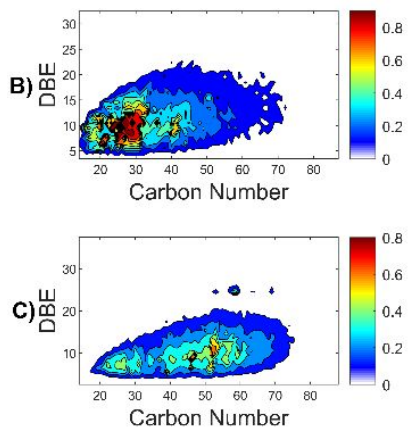

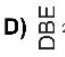

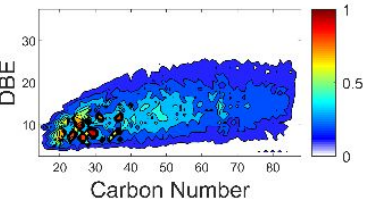

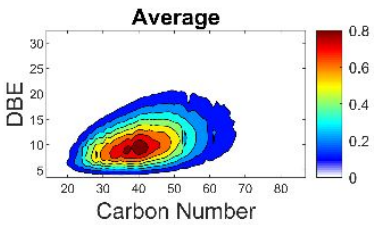
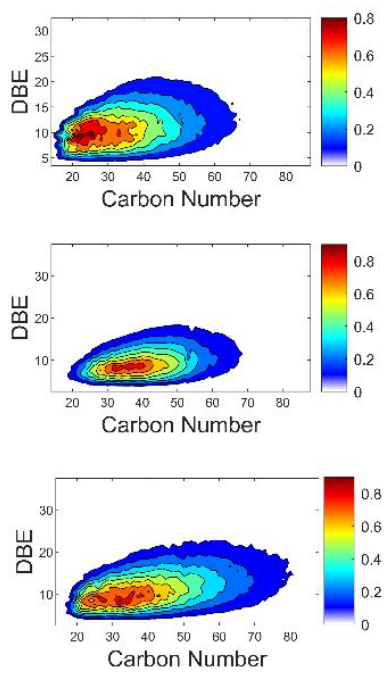
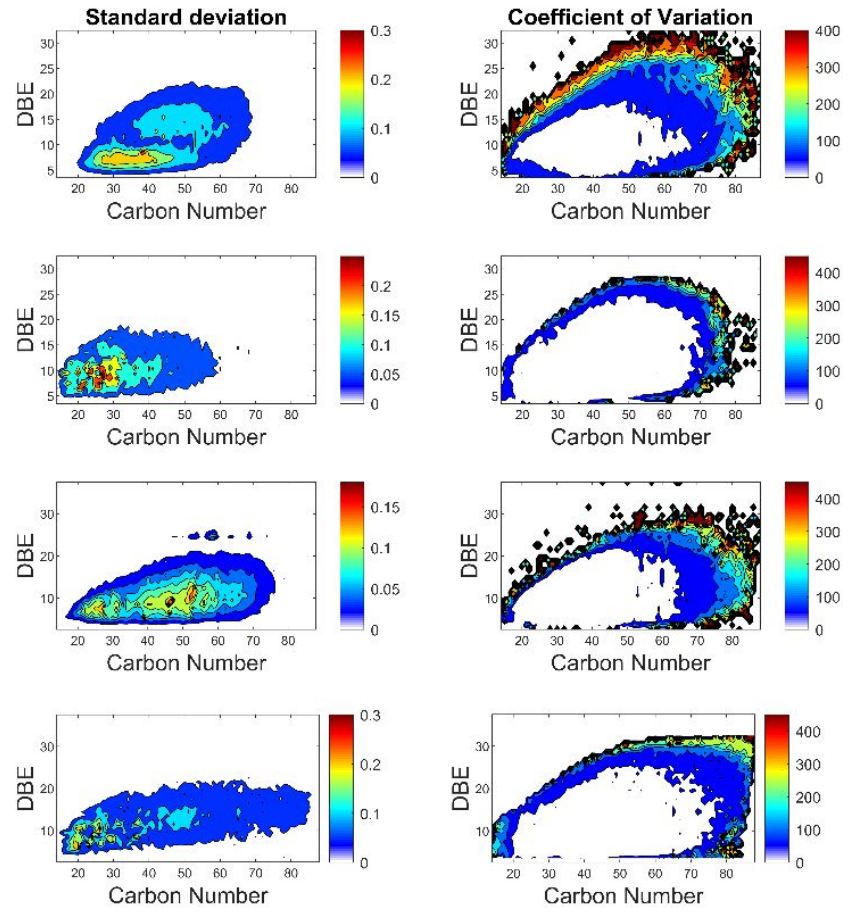

Figure S7. DBE versus $\mathrm{CN}$ plot of the majority class $\mathrm{N}[\mathrm{H}]$ in accordance with the range, mean, standard deviation, and $C V$ for a $n=21$ for Crude oils $C(A, B)$ and $D(C, D)$ by $E S I(+) F T-$ ICR MS $(A, C)$ and ESI(+)Orbitrap MS (B, D). 

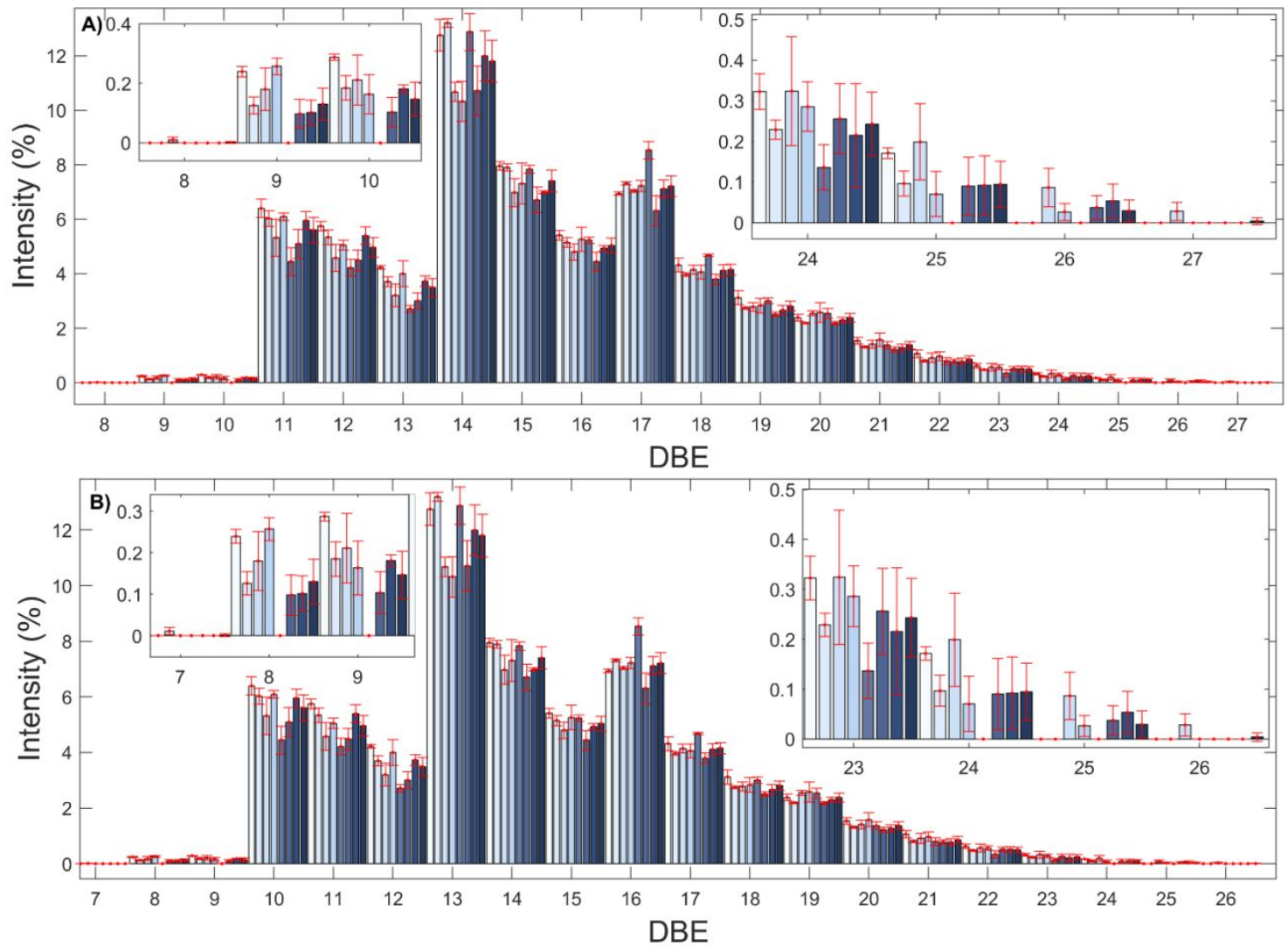

Figure S8. Plot of DBE distribution intensities of the majority class $\mathrm{N}[\mathrm{H}]$ by $(\mathrm{A}) \mathrm{ESI}(-) \mathrm{FT}-$ ICR MS and (B) ESI(-)Orbitrap MS for the averages $(n=3)$ for each day of analysis for Crude oil A. 

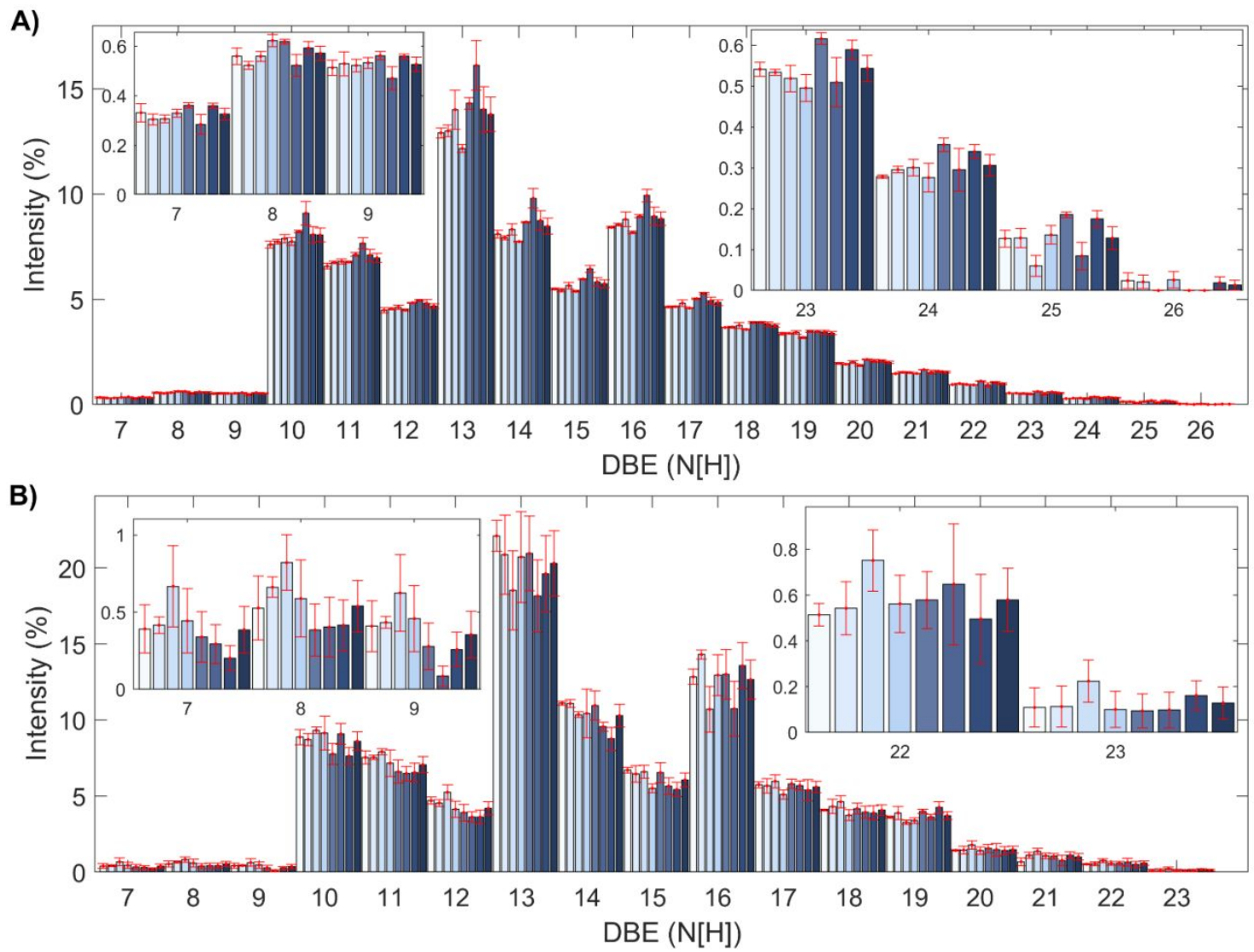

Figure S9. Plot of DBE distribution intensities of the majority class $\mathrm{N}[\mathrm{H}]$ by $(\mathrm{A}) \mathrm{ESI}(-) \mathrm{FT}-$ ICR MS and (B) ESI(-)Orbitrap MS for the replicate averages for each day of analysis for Crude oil B. 

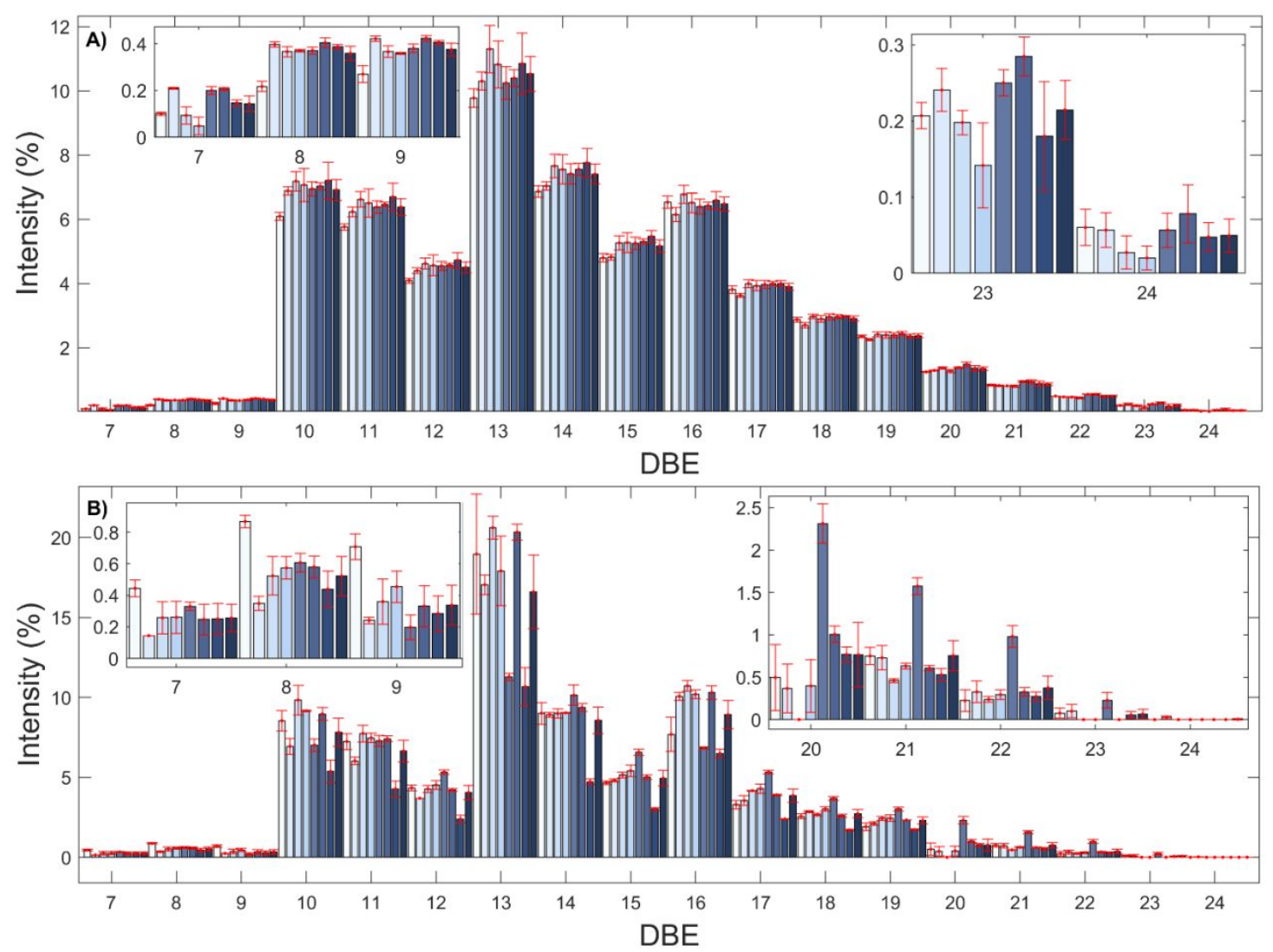

Figure S10. Plot of DBE distribution intensities of the majority class $\mathrm{N}[\mathrm{H}]$ by $(\mathrm{A}) \mathrm{ESI}(-) \mathrm{FT}-$ ICR MS and (B) ESI(-)Orbitrap MS for the averages $(n=3)$ for each day of analysis for Crude oil C. 

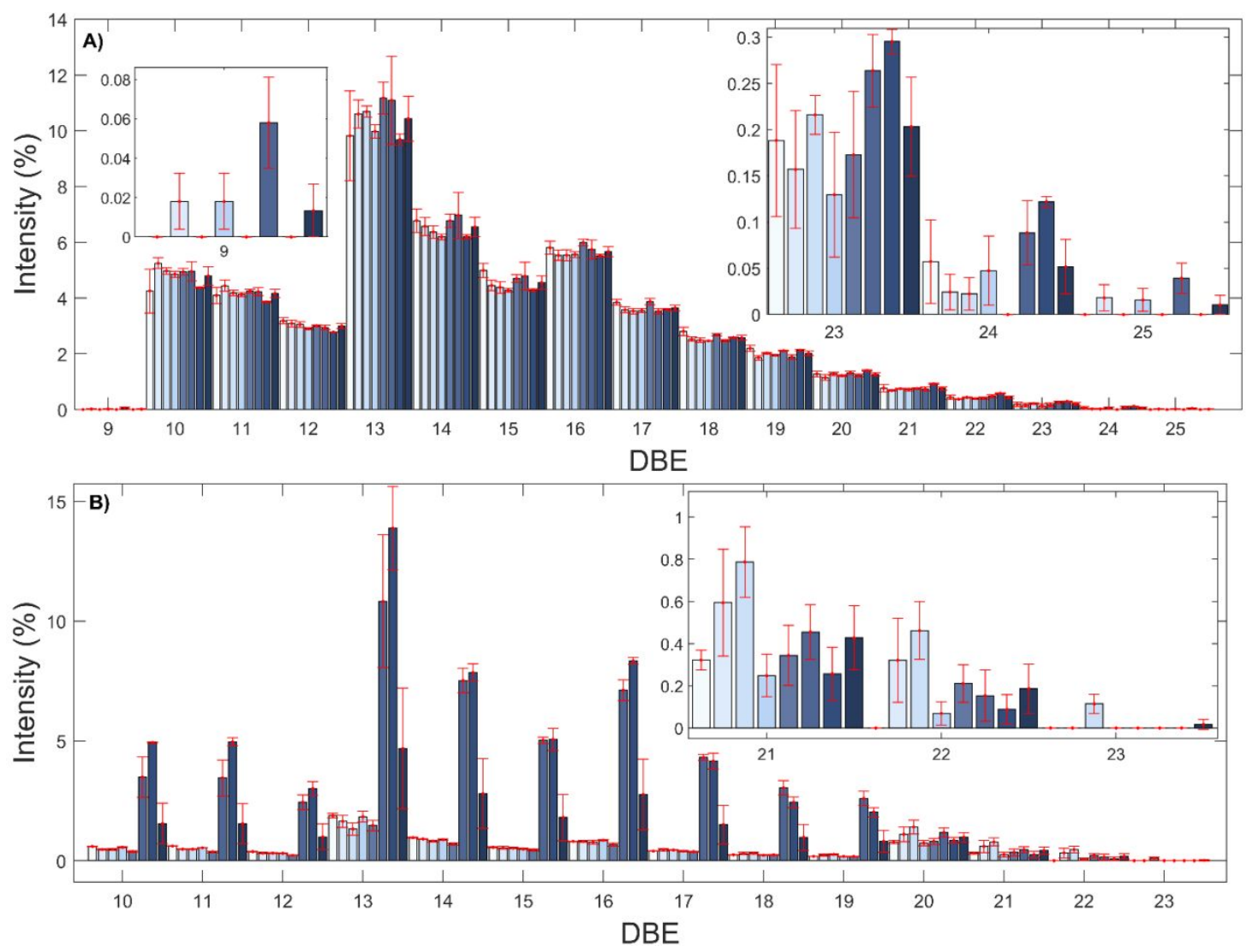

Figure S11. Plot of DBE distribution intensities of the majority class $\mathrm{N}[\mathrm{H}]$ by $(\mathrm{A}) \mathrm{ESI}(-) \mathrm{FT}-$ ICR MS and (B) ESI(-)Orbitrap MS for the averages $(n=3)$ for each day of analysis for Crude oil D. 


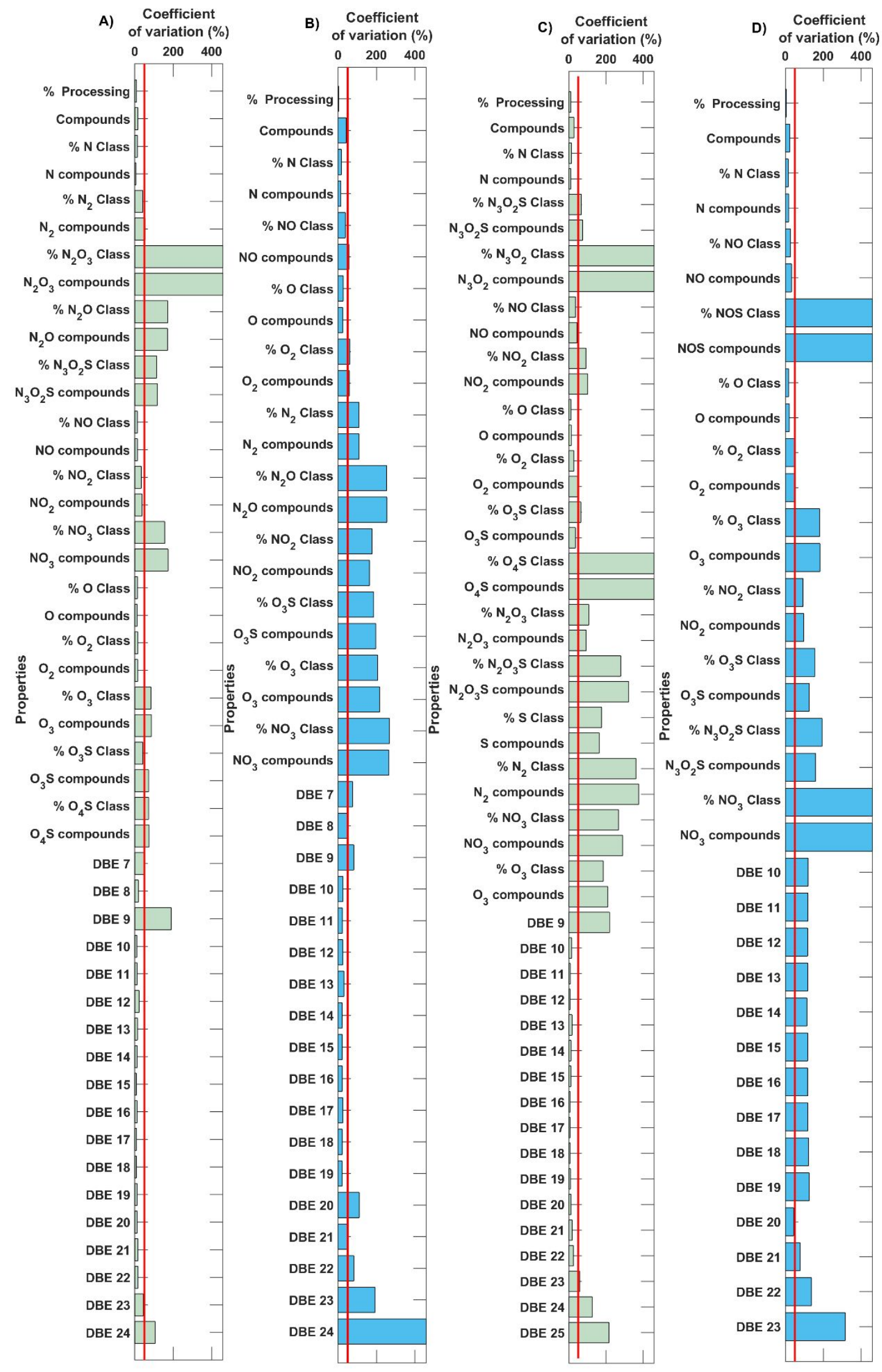

Figure S12. Plot of CV values of 21 analyzes by $(A, C)$ ESI(-)FT-ICR MS (Green bar) MS and $(B, D) E S I(+)$ Orbitrap MS (Blue bar) for Crude oil $C(A, B)$ and $D(C, D)$. 
A)

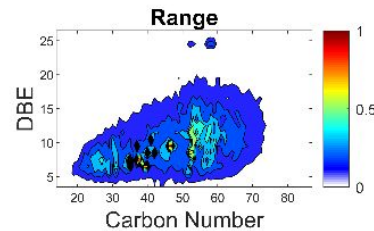

B)

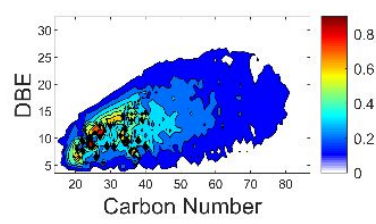

c) $\stackrel{\mathscr{m}}{\circ}$
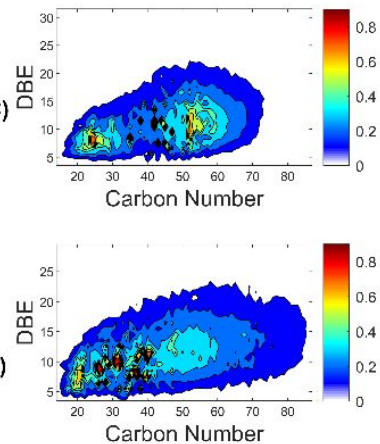
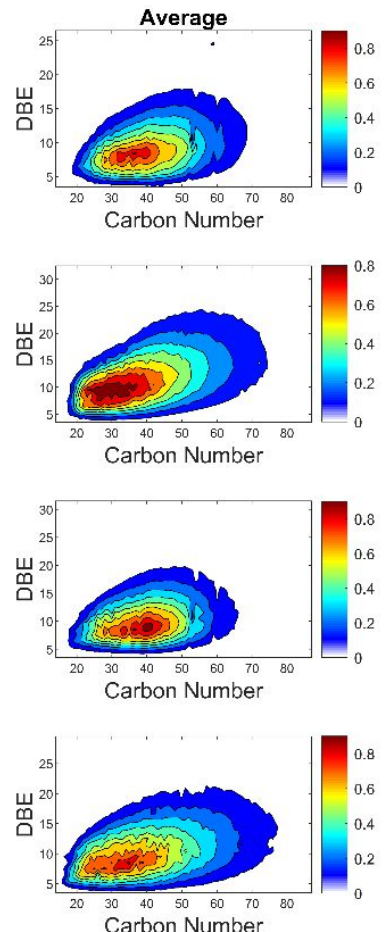
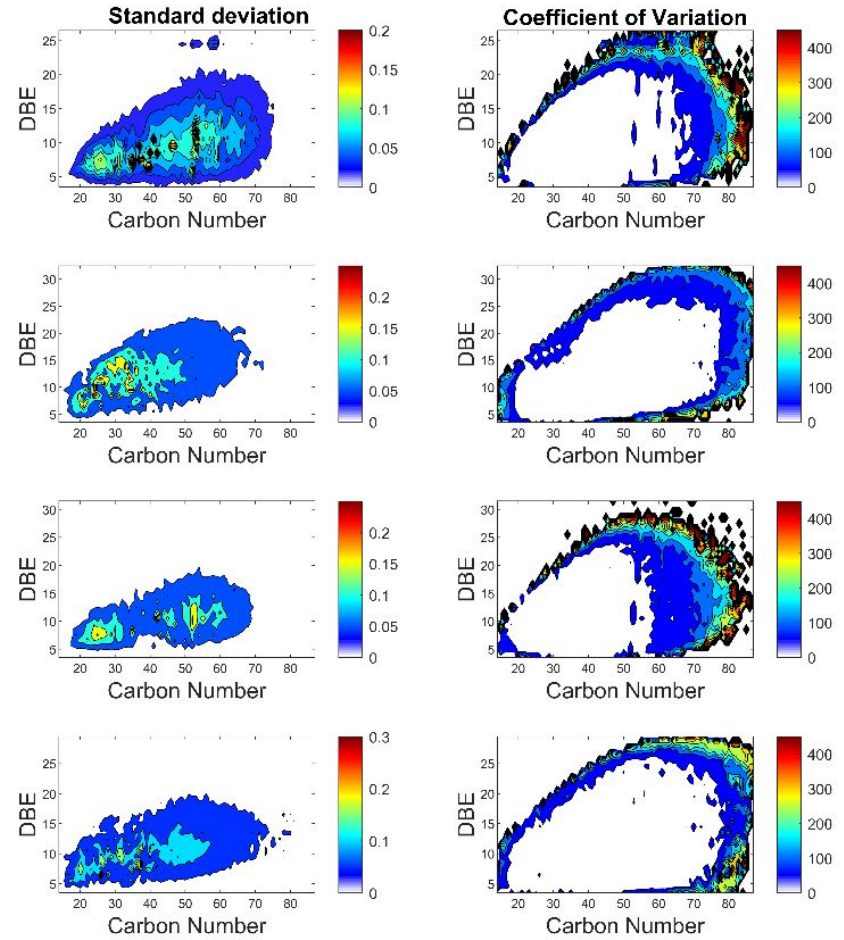

Figure S13. DBE versus $\mathrm{CN}$ plot of the majority class $\mathrm{N}[\mathrm{H}]$ in accordance with the range, mean, standard deviation, and CV for a $n=21$ for Crude oils $C(A, B)$ and $D(C, D)$ by ESI(-)FTICR MS (A,C) and ESI(-) Orbitrap MS (B, D).
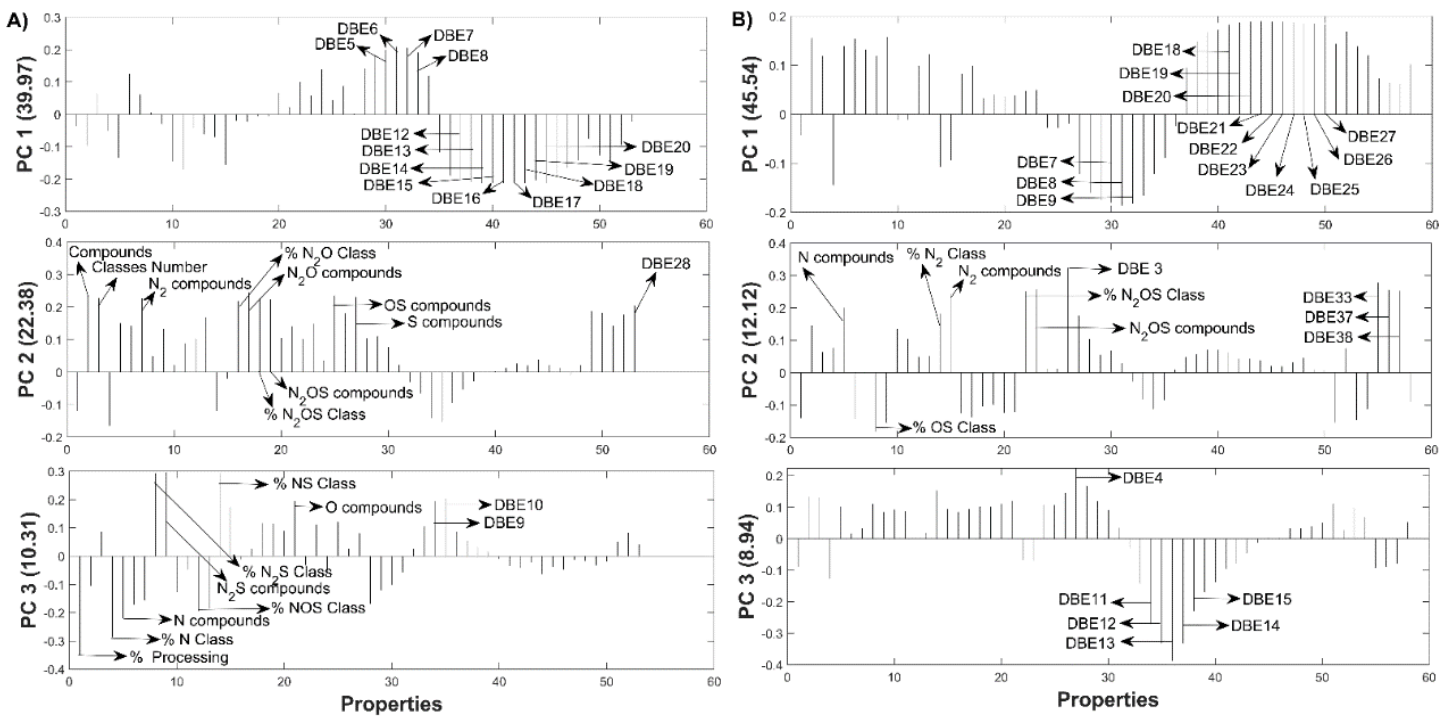

Figure S14. PCA loadings plot for Crude oils $A, B, C$, and $D$ analyzed by $(A) E S I(+) F T-$ ICR MS and (B) ESI(+)Orbitrap MS. 

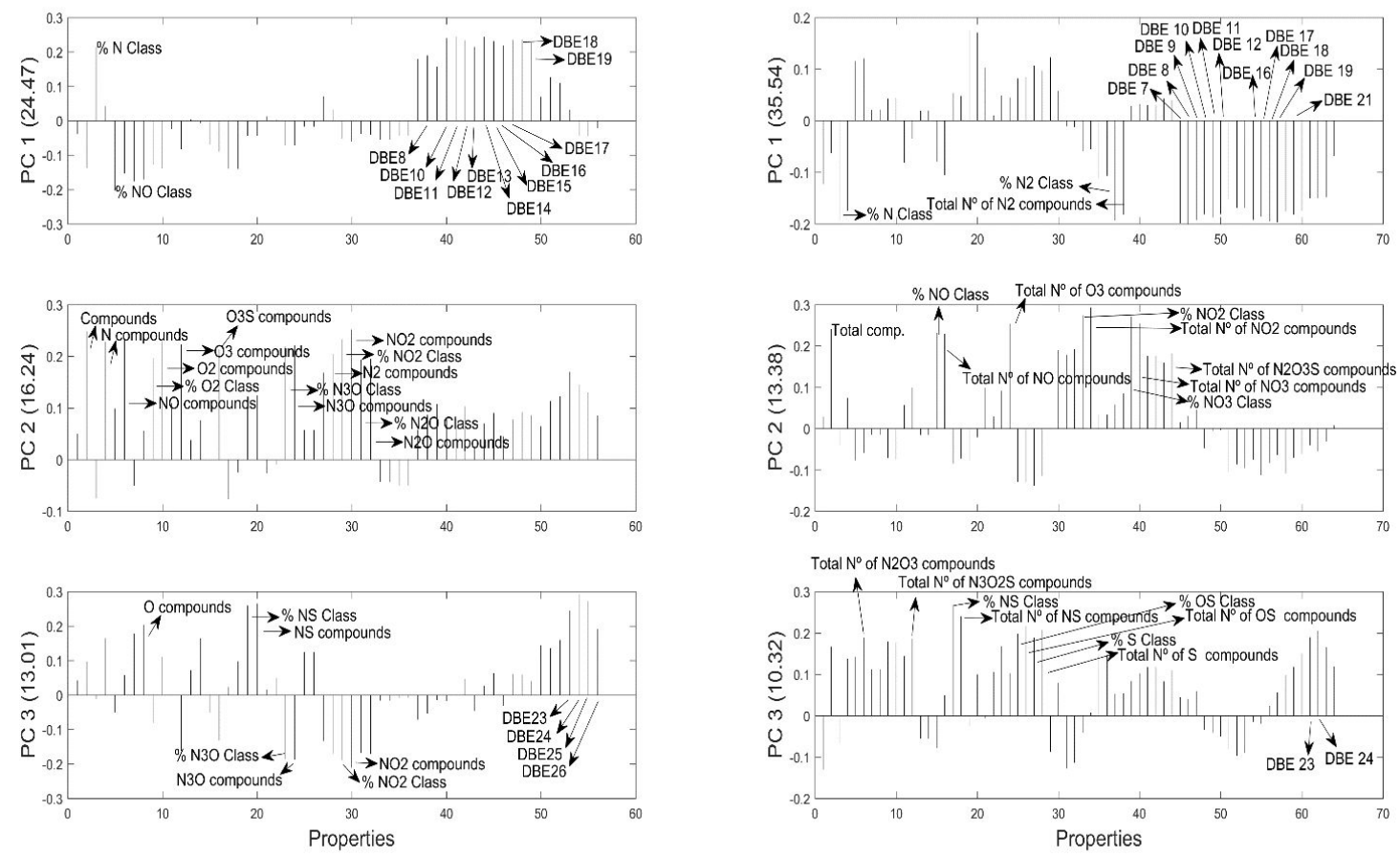

Figure S15. PCA loadings plot for Crude oils A, B, C, and D analyzed by (A) ESI(-)FT-ICR MS and (B) ESI(-)Orbitrap MS.

Table S1. Parameters used in the acquisition of crude oil data using ESI( \pm FT-ICR MS.

\begin{tabular}{ccc}
\hline Parameters & ESI(+) & ESI(-) \\
\hline Concentration $\left(\mathrm{mg} \mathrm{mL}^{-1}\right)$ & $1.5-2.0$ & $1.5-2.0$ \\
\hline Drying gas Flow $(\mathrm{L} \mathrm{min}-1)$ & 4 & 4 \\
\hline Capillary voltage $(\mathrm{kV})$ & 4.2 & $3.6-3.8$ \\
\hline Ion source gas temperature $\left({ }^{\circ} \mathrm{C}\right)$ & 250 & 180 \\
\hline Ion source gas pressure $($ bar $)$ & 1.0 & 1.0 \\
\hline Skimmer $(\mathrm{V})$ & 30.0 to 35.0 & $(-20)$ to $(-40)$ \\
\hline Collision voltage $(\mathrm{V})$ & $(-12)$ to $(-15)$ & 10 to 25 \\
\hline Ion Accumulation Time $(\mathrm{sec})$ & 0.12 to 0.15 & 0.070 to 0.080 \\
\hline Resolving Power & 718,937 to 946,859 & 515,762 to 756,737 \\
\hline
\end{tabular}


Table S2. Parameters used in the crude oil analyzes by ESI( \pm )Orbitrap MS data.

\begin{tabular}{ccc}
\hline Parameters & ESI(+) & ESI(-) \\
\hline Concentration $\left(\mathrm{mg} \mathrm{mL}^{-1}\right)$ & 0.1 & 0.1 \\
\hline Drying gas Flow $\left(\mathrm{L} \mathrm{min}^{-1}\right)$ & 2.5 & 2.5 \\
\hline Capillary voltage $(\mathrm{kV})$ & 3.5 & 3.85 \\
\hline lon source gas temperature $\left.{ }^{\circ} \mathrm{C}\right)$ & 275 & 275 \\
\hline Source ionization $(\mathrm{eV})$ & 45 & 45 \\
\hline tube lens $(\mathrm{V})$ & 80 & 80 \\
\hline AGC target & $5 \times 10^{6}$ & $5 \times 10^{6}$ \\
\hline Accumulation $(\mathrm{scan})$ & $10 \mu$ & 50 \\
\hline Capilary temp (C) & 275 & 250 \\
\hline Flow rate $\left(\mu L \mathrm{~min}^{-1}\right)$ & 2.50 & 2.00 \\
\hline Acquisition interval $(\mathrm{m} / \mathrm{z})$ & $200-1.200$ & $150-1.000$ \\
\hline Resolving Power & \multirow{2}{*}{$87.001-144.303$} & $91.700-$ \\
& & 169.4020
\end{tabular}

Table S3. Parameters for mass spectra processing in the Composer ${ }^{\circledR}$ software for $\mathrm{ESI}(+)$ and ESI(-) to FT-ICR and Orbitrap MS data.

\begin{tabular}{ccc}
\hline Parameters & ESI(-) & ESI(+) \\
\hline Filter spectrum by S/N (\%) & - & - \\
\hline Recalibration method & Homologous series & Homologous series \\
\hline Recalibration equation & Walking Recalibration & Walking Recalibration \\
\hline Tolerance window (ppm) & 5.00 & 5.00 \\
\hline Intensity Threshold (\%) & 2.50 & 2.50 \\
\hline Ion properties & Allow adduct/loss ions & Allow adduct/loss ions only \\
\hline Range (Da) & $200-800$ & $200-1000$ \\
\hline m/z match tolerance (ppm) & 1 to FT-ICR MS & 1 to FT-ICR MS \\
\hline Minimum abundance (\%) & 1 & Orbitrap MS Orbitrap MS \\
\hline Computation mode & All compounds & All compounds \\
\hline De novo m/z upper limit (Da) & 800 & 1000 \\
\hline Minimum de novo abundance & 0 & 0 \\
\hline Element ranges & $S=1 ; 0=4 ; N=4$ & $S=1 ; 0=3 ; N=3$ \\
\hline
\end{tabular}


Table S4. Example of matrix input of variables for building the PCA model (Part 1).

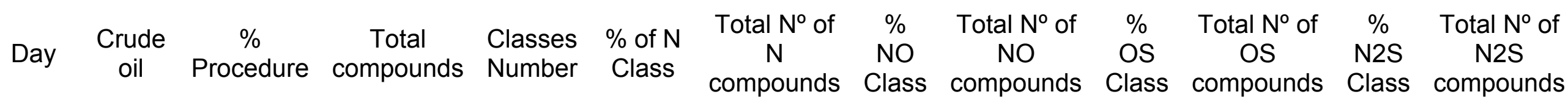

\begin{tabular}{|c|c|c|c|c|c|c|c|c|c|c|c|c|}
\hline 1 & 1 & 90.73 & 1404.00 & 3.00 & 85.83 & 1025.00 & 4.24 & 325.00 & 0.66 & 55.00 & 0.00 & 0.00 \\
\hline 1 & 1 & 94.00 & 2458.00 & 5.00 & 82.56 & 1378.00 & 8.69 & 787.00 & 1.51 & 185.00 & 0.20 & 45.00 \\
\hline 1 & 1 & 96.18 & 2597.00 & 5.00 & 85.90 & 1530.00 & 8.41 & 823.00 & 1.69 & 202.00 & 0.00 & 0.00 \\
\hline 2 & 1 & 94.87 & 2321.00 & 4.00 & 77.28 & 1339.00 & 10.93 & 697.00 & 6.46 & 262.00 & 0.21 & 21.00 \\
\hline 2 & 1 & 94.35 & 2421.00 & 5.00 & 72.23 & 1348.00 & 12.09 & 771.00 & 9.92 & 347.00 & 0.00 & 0.00 \\
\hline 2 & 1 & 95.51 & 2552.00 & 5.00 & 83.02 & 1501.00 & 9.24 & 769.00 & 2.94 & 224.00 & 0.00 & 0.00 \\
\hline 3 & 1 & 95.28 & 2591.00 & 7.00 & 83.78 & 1469.00 & 8.41 & 801.00 & 2.70 & 214.00 & 0.09 & 15.00 \\
\hline 3 & 1 & 95.34 & 2681.00 & 7.00 & 83.70 & 1485.00 & 8.50 & 831.00 & 2.57 & 215.00 & 0.19 & 27.00 \\
\hline 3 & 1 & 95.60 & 2636.00 & 7.00 & 83.92 & 1473.00 & 8.56 & 827.00 & 2.67 & 222.00 & 0.20 & 29.00 \\
\hline 4 & 1 & 96.45 & 2053.00 & 6.00 & 89.12 & 1240.00 & 5.73 & 561.00 & 0.97 & 127.00 & 0.24 & 43.00 \\
\hline 4 & 1 & 98.48 & 1903.00 & 7.00 & 87.93 & 1182.00 & 4.62 & 497.00 & 0.66 & 88.00 & 0.33 & 57.00 \\
\hline 4 & 1 & 96.71 & 1906.00 & 5.00 & 92.23 & 1262.00 & 3.64 & 461.00 & 0.19 & 47.00 & 0.23 & 44.00 \\
\hline 5 & 1 & 98.37 & 2259.00 & 7.00 & 89.41 & 1366.00 & 6.98 & 676.00 & 1.35 & 143.00 & 0.13 & 21.00 \\
\hline 5 & 1 & 98.89 & 2604.00 & 6.00 & 80.39 & 1398.00 & 10.41 & 773.00 & 6.56 & 320.00 & 0.00 & 0.00 \\
\hline 5 & 1 & 93.34 & 2503.00 & 9.00 & 80.14 & 1335.00 & 9.71 & 786.00 & 2.57 & 199.00 & 0.53 & 75.00 \\
\hline 6 & 1 & 96.55 & 2085.00 & 7.00 & 88.91 & 1236.00 & 5.89 & 579.00 & 1.02 & 125.00 & 0.40 & 72.00 \\
\hline 6 & 1 & 95.11 & 2113.00 & 5.00 & 88.70 & 1282.00 & 4.40 & 534.00 & 0.10 & 22.00 & 0.46 & 64.00 \\
\hline 6 & 1 & 94.36 & 2603.00 & 7.00 & 82.31 & 1395.00 & 8.34 & 794.00 & 2.69 & 228.00 & 0.76 & 128.00 \\
\hline 7 & 1 & 96.55 & 2085.00 & 5.00 & 88.91 & 1236.00 & 5.89 & 579.00 & 1.02 & 125.00 & 0.40 & 72.00 \\
\hline 7 & 1 & 95.11 & 2113.00 & 5.00 & 88.70 & 1282.00 & 4.40 & 534.00 & 0.10 & 22.00 & 0.46 & 64.00 \\
\hline 7 & 1 & 94.36 & 2603.00 & 7.00 & 82.31 & 1395.00 & 8.34 & 794.00 & 2.69 & 228.00 & 0.76 & 128.00 \\
\hline 1 & 2 & 93.84 & 2150.00 & 5.00 & 87.52 & 1391.00 & 5.40 & 595.00 & 0.04 & 10.00 & 0.00 & 0.00 \\
\hline 1 & 2 & 95.15 & 1886.00 & 4.00 & 89.49 & 1133.00 & 4.10 & 531.00 & 0.00 & 0.00 & 0.00 & 0.00 \\
\hline 1 & 2 & 94.49 & 2171.00 & 4.00 & 89.66 & 1410.00 & 3.06 & 484.00 & 0.00 & 0.00 & 0.43 & 55.00 \\
\hline 2 & 2 & 95.13 & 2602.00 & 5.00 & 86.51 & 1507.00 & 5.87 & 662.00 & 0.29 & 82.00 & 0.70 & 98.00 \\
\hline
\end{tabular}




\begin{tabular}{|c|c|c|c|c|c|c|c|c|c|c|c|c|}
\hline 2 & 2 & 95.37 & 2620.00 & 6.00 & 86.63 & 1500.00 & 5.86 & 665.00 & 0.03 & 84.00 & 0.59 & 57.00 \\
\hline 2 & 2 & 94.55 & 2155.00 & 4.00 & 88.70 & 1318.00 & 3.13 & 479.00 & 0.00 & 0.00 & 0.58 & 68.00 \\
\hline 3 & 2 & 93.54 & 3050.00 & 7.00 & 84.61 & 1698.00 & 6.69 & 824.00 & 0.39 & 125.00 & 0.68 & 86.00 \\
\hline 3 & 2 & 93.12 & 2739.00 & 7.00 & 84.64 & 1566.00 & 6.06 & 717.00 & 0.23 & 62.00 & 0.82 & 107.00 \\
\hline 3 & 2 & 93.74 & 2723.00 & 7.00 & 86.36 & 1614.00 & 5.76 & 720.00 & 0.20 & 77.00 & 0.35 & 46.00 \\
\hline 4 & 2 & 94.10 & 1890.00 & 4.00 & 87.93 & 1102.00 & 1.78 & 321.00 & 0.00 & 0.00 & 1.67 & 199.00 \\
\hline 4 & 2 & 94.92 & 1947.00 & 5.00 & 89.57 & 1229.00 & 2.67 & 399.00 & 0.02 & 6.00 & 0.50 & 79.00 \\
\hline 4 & 2 & 94.66 & 1993.00 & 4.00 & 88.45 & 1174.00 & 2.76 & 415.00 & 0.00 & 0.00 & 1.32 & 171.00 \\
\hline 5 & 2 & 95.52 & 2046.00 & 5.00 & 89.03 & 1237.00 & 3.13 & 428.00 & 0.00 & 0.00 & 0.10 & 10.00 \\
\hline 5 & 2 & 95.30 & 2383.00 & 5.00 & 88.28 & 1427.00 & 4.42 & 567.00 & 0.08 & 31.00 & 0.68 & 109.00 \\
\hline 5 & 2 & 94.08 & 1745.00 & 4.00 & 89.15 & 1208.00 & 2.76 & 304.00 & 0.00 & 0.00 & 0.83 & 74.00 \\
\hline 6 & 2 & 94.72 & 2575.00 & 6.00 & 85.96 & 1469.00 & 5.81 & 652.00 & 0.32 & 90.00 & 0.54 & 66.00 \\
\hline 6 & 2 & 95.13 & 2602.00 & 5.00 & 86.51 & 1507.00 & 5.87 & 662.00 & 0.29 & 82.00 & 0.70 & 98.00 \\
\hline 6 & 2 & 94.83 & 2542.00 & 5.00 & 86.20 & 1468.00 & 5.73 & 642.00 & 0.33 & 84.00 & 0.68 & 74.00 \\
\hline 7 & 2 & 94.72 & 2575.00 & 6.00 & 85.96 & 1469.00 & 5.81 & 652.00 & 0.32 & 90.00 & 0.54 & 66.00 \\
\hline 7 & 2 & 95.13 & 2602.00 & 5.00 & 86.51 & 1507.00 & 5.87 & 662.00 & 0.29 & 82.00 & 0.70 & 98.00 \\
\hline 7 & 2 & 94.83 & 2542.00 & 5.00 & 86.20 & 1468.00 & 5.73 & 642.00 & 0.33 & 84.00 & 0.68 & 74.00 \\
\hline 1 & 3 & 94.72 & 1977.00 & 4.00 & 89.70 & 1285.00 & 3.47 & 472.00 & 0.00 & 0.00 & 0.20 & 28.00 \\
\hline 1 & 3 & 95.78 & 2041.00 & 5.00 & 90.58 & 1276.00 & 4.02 & 551.00 & 0.00 & 0.00 & 0.06 & 10.00 \\
\hline 1 & 3 & 95.76 & 2145.00 & 5.00 & 90.32 & 1365.00 & 3.96 & 533.00 & 0.03 & 5.00 & 0.33 & 49.00 \\
\hline 2 & 3 & 94.70 & 1973.00 & 5.00 & 89.05 & 1217.00 & 2.86 & 419.00 & 0.06 & 6.00 & 0.90 & 120.00 \\
\hline 2 & 3 & 94.99 & 2049.00 & 4.00 & 89.36 & 1265.00 & 3.31 & 468.00 & 0.00 & 0.00 & 0.35 & 51.00 \\
\hline 2 & 3 & 95.53 & 2392.00 & 6.00 & 89.90 & 1512.00 & 4.34 & 617.00 & 0.00 & 5.00 & 0.14 & 21.00 \\
\hline 3 & 3 & 95.28 & 2277.00 & 5.00 & 87.81 & 1332.00 & 4.72 & 570.00 & 0.04 & 6.00 & 0.99 & 142.00 \\
\hline 3 & 3 & 94.95 & 2384.00 & 7.00 & 89.33 & 1479.00 & 4.45 & 628.00 & 0.03 & 6.00 & 0.26 & 38.00 \\
\hline 3 & 3 & 95.15 & 2395.00 & 7.00 & 89.09 & 1428.00 & 4.14 & 588.00 & 0.03 & 6.00 & 0.48 & 54.00 \\
\hline 4 & 3 & 94.86 & 1921.00 & 6.00 & 89.84 & 1215.00 & 2.89 & 418.00 & 0.16 & 26.00 & 0.35 & 59.00 \\
\hline 4 & 3 & 93.95 & 1829.00 & 5.00 & 88.37 & 1120.00 & 2.48 & 370.00 & 0.07 & 6.00 & 0.06 & 10.00 \\
\hline 4 & 3 & 94.77 & 1847.00 & 5.00 & 89.16 & 1139.00 & 2.60 & 379.00 & 0.11 & 7.00 & 0.61 & 99.00 \\
\hline 5 & 3 & 98.19 & 2176.00 & 5.00 & 88.05 & 1239.00 & 4.81 & 555.00 & 0.25 & 64.00 & 0.20 & 29.00 \\
\hline 5 & 3 & 95.11 & 2113.00 & 5.00 & 88.70 & 1282.00 & 4.40 & 534.00 & 0.10 & 22.00 & 0.46 & 64.00 \\
\hline 5 & 3 & 95.28 & 2190.00 & 5.00 & 87.99 & 1263.00 & 4.55 & 560.00 & 0.05 & 6.00 & 0.98 & 110.00 \\
\hline
\end{tabular}




\begin{tabular}{|c|c|c|c|c|c|c|c|c|c|c|c|c|}
\hline 6 & 3 & 95.42 & 2207.00 & 5.00 & 89.02 & 1338.00 & 4.34 & 532.00 & 0.14 & 35.00 & 0.60 & 97.00 \\
\hline 6 & 3 & 95.63 & 2016.00 & 4.00 & 90.74 & 1279.00 & 3.82 & 534.00 & 0.00 & 0.00 & 0.00 & 0.00 \\
\hline 6 & 3 & 93.55 & 1816.00 & 5.00 & 88.30 & 1142.00 & 2.47 & 369.00 & 0.06 & 5.00 & 0.60 & 90.00 \\
\hline 7 & 3 & 95.42 & 2207.00 & 5.00 & 89.02 & 1338.00 & 4.34 & 532.00 & 0.14 & 35.00 & 0.60 & 97.00 \\
\hline 7 & 3 & 95.63 & 2016.00 & 4.00 & 90.74 & 1279.00 & 3.82 & 534.00 & 0.00 & 0.00 & 0.00 & 0.00 \\
\hline 7 & 3 & 93.55 & 1816.00 & 5.00 & 88.30 & 1142.00 & 2.47 & 369.00 & 0.06 & 5.00 & 0.60 & 90.00 \\
\hline 1 & 4 & 97.13 & 1647.00 & 3.00 & 92.15 & 1077.00 & 4.45 & 1647.00 & 0.00 & 0.00 & 0.00 & 0.00 \\
\hline 1 & 4 & 96.90 & 1289.00 & 3.00 & 94.31 & 958.00 & 1.94 & 225.00 & 0.00 & 0.00 & 0.00 & 0.00 \\
\hline 1 & 4 & 97.37 & 1475.00 & 3.00 & 93.87 & 1022.00 & 2.81 & 335.00 & 0.00 & 0.00 & 0.00 & 0.00 \\
\hline 2 & 4 & 96.08 & 1730.00 & 4.00 & 91.11 & 1096.00 & 3.62 & 395.00 & 0.00 & 0.00 & 0.71 & 118.00 \\
\hline 2 & 4 & 96.31 & 1787.00 & 4.00 & 90.95 & 1100.00 & 3.83 & 418.00 & 0.00 & 0.00 & 0.81 & 130.00 \\
\hline 2 & 4 & 93.31 & 1913.00 & 5.00 & 86.56 & 1167.00 & 5.12 & 475.00 & 0.07 & 21.00 & 0.78 & 116.00 \\
\hline 3 & 4 & 92.73 & 2232.00 & 5.00 & 73.33 & 1180.00 & 13.57 & 736.00 & 5.77 & 308.00 & 0.00 & 0.00 \\
\hline 3 & 4 & 95.70 & 1959.00 & 5.00 & 85.91 & 1096.00 & 7.73 & 543.00 & 0.65 & 95.00 & 0.61 & 100.00 \\
\hline 3 & 4 & 96.13 & 2326.00 & 5.00 & 83.05 & 1200.00 & 9.86 & 708.00 & 2.03 & 193.00 & 0.62 & 114.00 \\
\hline 4 & 4 & 96.91 & 1347.00 & 4.00 & 92.66 & 941.00 & 3.26 & 273.00 & 0.00 & 0.00 & 0.11 & 17.00 \\
\hline 4 & 4 & 98.43 & 1339.00 & 4.00 & 93.18 & 945.00 & 2.72 & 257.00 & 0.00 & 0.00 & 0.06 & 10.00 \\
\hline 4 & 4 & 96.00 & 1373.00 & 4.00 & 91.91 & 986.00 & 3.37 & 290.00 & 0.00 & 0.00 & 0.03 & 5.00 \\
\hline 5 & 4 & 95.77 & 2137.00 & 7.00 & 81.51 & 1090.00 & 10.45 & 646.00 & 2.54 & 197.00 & 0.78 & 123.00 \\
\hline 5 & 4 & 95.88 & 1983.00 & 5.00 & 85.26 & 1064.00 & 8.29 & 573.00 & 0.63 & 92.00 & 1.08 & 154.00 \\
\hline 5 & 4 & 95.55 & 1850.00 & 5.00 & 87.03 & 1057.00 & 6.53 & 491.00 & 0.48 & 73.00 & 0.85 & 125.00 \\
\hline 6 & 4 & 96.65 & 1625.00 & 3.00 & 91.64 & 1071.00 & 4.42 & 436.00 & 0.00 & 0.00 & 0.00 & 0.00 \\
\hline 6 & 4 & 95.52 & 1957.00 & 5.00 & 85.58 & 1120.00 & 8.11 & 556.00 & 0.70 & 103.00 & 0.29 & 50.00 \\
\hline 6 & 4 & 95.73 & 1349.00 & 4.00 & 91.27 & 959.00 & 3.71 & 294.00 & 0.00 & 0.00 & 0.06 & 10.00 \\
\hline 7 & 4 & 96.65 & 1625.00 & 3.00 & 91.64 & 1071.00 & 4.42 & 436.00 & 0.00 & 0.00 & 0.00 & 0.00 \\
\hline 7 & 4 & 95.52 & 1957.00 & 5.00 & 85.58 & 1120.00 & 8.11 & 556.00 & 0.70 & 103.00 & 0.29 & 50.00 \\
\hline 7 & 4 & 95.73 & 1349.00 & 4.00 & 91.27 & 959.00 & 3.71 & 294.00 & 0.00 & 0.00 & 0.06 & 10.00 \\
\hline
\end{tabular}


Table S5. Example of matrix input of variables for building the PCA model (Part 2).

Crude \% Total $\mathrm{N}^{\circ}$ of oil $\quad$ NS $\quad$ NS $\quad$ Class
Total $\mathrm{N}^{\circ}$ of $\% \quad$ Total $\mathrm{N}^{\circ}$ of $\mathrm{N}_{2}$ compounds Class compounds
$\% \mathrm{O}$ Class $\begin{array}{lllll}\text { Total } \mathrm{N}^{\circ} \text { of } & \% & \text { Total } & \mathrm{N}^{\circ} \text { of } & \%\end{array} \quad$ Total $\mathrm{N}^{\circ}$ of

$\begin{array}{lllll}\mathrm{O} & \mathrm{HC} & \mathrm{HC} & \mathrm{N}_{2} \mathrm{OS} & \mathrm{N}_{2} \mathrm{OS}\end{array}$ compounds Class compounds Class compounds

\begin{tabular}{|c|c|c|c|c|c|c|c|c|c|c|c|c|c|}
\hline 1 & 1 & 0.00 & 0.00 & 0.00 & 0.00 & 0.00 & 0.00 & 0.00 & 0.00 & 0.00 & 0.00 & 0.00 & 0.00 \\
\hline 1 & 1 & 1.00 & 63.00 & 0.00 & 0.00 & 0.00 & 0.00 & 0.00 & 0.00 & 0.00 & 0.00 & 0.00 & 0.00 \\
\hline 1 & 1 & 0.12 & 16.00 & 0.07 & 26.00 & 0.00 & 0.00 & 0.00 & 0.00 & 0.00 & 0.00 & 0.00 & 0.00 \\
\hline 2 & 1 & 0.00 & 0.00 & 0.00 & 0.00 & 0.00 & 0.00 & 0.00 & 0.00 & 0.00 & 0.00 & 0.00 & 0.00 \\
\hline 2 & 1 & 0.07 & 6.00 & 0.00 & 0.00 & 0.04 & 8.00 & 0.00 & 0.00 & 0.00 & 0.00 & 0.00 & 0.00 \\
\hline 2 & 1 & 0.17 & 16.00 & 0.00 & 0.00 & 0.13 & 40.00 & 0.00 & 0.00 & 0.00 & 0.00 & 0.00 & 0.00 \\
\hline 3 & 1 & 0.02 & 5.00 & 0.11 & 32.00 & 0.16 & 54.00 & 0.00 & 0.00 & 0.00 & 0.00 & 0.00 & 0.00 \\
\hline 3 & 1 & 0.09 & 12.00 & 0.08 & 32.00 & 0.21 & 72.00 & 0.00 & 0.00 & 0.00 & 0.00 & 0.00 & 0.00 \\
\hline 3 & 1 & 0.05 & 10.00 & 0.07 & 29.00 & 0.12 & 45.00 & 0.00 & 0.00 & 0.00 & 0.00 & 0.00 & 0.00 \\
\hline 4 & 1 & 0.03 & 6.00 & 0.36 & 76.00 & 0.00 & 0.00 & 0.00 & 0.00 & 0.00 & 0.00 & 0.00 & 0.00 \\
\hline 4 & 1 & 0.02 & 5.00 & 0.35 & 69.00 & 0.04 & 5.00 & 0.00 & 0.00 & 0.00 & 0.00 & 0.00 & 0.00 \\
\hline 4 & 1 & 0.00 & 0.00 & 0.42 & 92.00 & 0.00 & 0.00 & 0.00 & 0.00 & 0.00 & 0.00 & 0.00 & 0.00 \\
\hline 5 & 1 & 0.05 & 10.00 & 0.14 & 36.00 & 0.00 & 0.00 & 0.02 & 7.00 & 0.00 & 0.00 & 0.00 & 0.00 \\
\hline 5 & 1 & 0.12 & 10.00 & 0.05 & 16.00 & 0.48 & 87.00 & 0.00 & 0.00 & 0.00 & 0.00 & 0.00 & 0.00 \\
\hline 5 & 1 & 0.11 & 22.00 & 0.14 & 41.00 & 0.09 & 30.00 & 0.03 & 8.00 & 0.03 & 7.00 & 0.00 & 0.00 \\
\hline 6 & 1 & 0.00 & 0.00 & 0.34 & 72.00 & 0.00 & 0.00 & 0.00 & 0.00 & 0.00 & 0.00 & 0.00 & 0.00 \\
\hline 6 & 1 & 0.00 & 0.00 & 1.45 & 211.00 & 0.00 & 0.00 & 0.00 & 0.00 & 0.00 & 0.00 & 0.00 & 0.00 \\
\hline 6 & 1 & 0.14 & 11.00 & 0.08 & 32.00 & 0.03 & 15.00 & 0.00 & 0.00 & 0.00 & 0.00 & 0.00 & 0.00 \\
\hline 7 & 1 & 0.00 & 0.00 & 0.34 & 73.00 & 0.00 & 0.00 & 0.00 & 0.00 & 0.00 & 0.00 & 0.00 & 0.00 \\
\hline 7 & 1 & 0.00 & 0.00 & 1.45 & 211.00 & 0.00 & 0.00 & 0.00 & 0.00 & 0.00 & 0.00 & 0.00 & 0.00 \\
\hline 7 & 1 & 0.14 & 11.00 & 0.08 & 32.00 & 0.03 & 15.00 & 0.00 & 0.00 & 0.00 & 0.00 & 0.00 & 0.00 \\
\hline 1 & 2 & 0.03 & 6.00 & 0.82 & 148.00 & 0.00 & 0.00 & 0.00 & 0.00 & 0.00 & 0.00 & 0.00 & 0.00 \\
\hline 1 & 2 & 0.02 & 5.00 & 1.53 & 216.00 & 0.00 & 0.00 & 0.00 & 0.00 & 0.00 & 0.00 & 0.00 & 0.00 \\
\hline 1 & 2 & 0.00 & 0.00 & 1.34 & 221.00 & 0.00 & 0.00 & 0.00 & 0.00 & 0.00 & 0.00 & 0.00 & 0.00 \\
\hline 2 & 2 & 0.00 & 0.00 & 1.76 & 257.00 & 0.00 & 0.00 & 0.00 & 0.00 & 0.00 & 0.00 & 0.00 & 0.00 \\
\hline
\end{tabular}




\begin{tabular}{|c|c|c|c|c|c|c|c|c|c|c|c|c|c|}
\hline 2 & 2 & 0.03 & 6.00 & 1.97 & 300.00 & 0.00 & 0.00 & 0.00 & 0.00 & 0.00 & 0.00 & 0.00 & 0.00 \\
\hline 2 & 2 & 0.00 & 0.00 & 2.14 & 290.00 & 0.00 & 0.00 & 0.00 & 0.00 & 0.00 & 0.00 & 0.00 & 0.00 \\
\hline 3 & 2 & 0.16 & 18.00 & 0.72 & 172.00 & 0.00 & 0.00 & 0.00 & 0.00 & 0.00 & 0.00 & 0.29 & 124.00 \\
\hline 3 & 2 & 0.43 & 29.00 & 0.79 & 186.00 & 0.00 & 0.00 & 0.00 & 0.00 & 0.00 & 0.00 & 0.15 & 72.00 \\
\hline 3 & 2 & 0.21 & 20.00 & 0.73 & 177.00 & 0.00 & 0.00 & 0.00 & 0.00 & 0.00 & 0.00 & 0.14 & 69.00 \\
\hline 4 & 2 & 0.00 & 0.00 & 2.72 & 268.00 & 0.00 & 0.00 & 0.00 & 0.00 & 0.00 & 0.00 & 0.00 & 0.00 \\
\hline 4 & 2 & 0.00 & 0.00 & 2.16 & 234.00 & 0.00 & 0.00 & 0.00 & 0.00 & 0.00 & 0.00 & 0.00 & 0.00 \\
\hline 4 & 2 & 0.00 & 0.00 & 2.13 & 233.00 & 0.00 & 0.00 & 0.00 & 0.00 & 0.00 & 0.00 & 0.00 & 0.00 \\
\hline 5 & 2 & 0.02 & 6.00 & 3.24 & 365.00 & 0.00 & 0.00 & 0.00 & 0.00 & 0.00 & 0.00 & 0.00 & 0.00 \\
\hline 5 & 2 & 0.00 & 0.00 & 1.83 & 249.00 & 0.00 & 0.00 & 0.00 & 0.00 & 0.00 & 0.00 & 0.00 & 0.00 \\
\hline 5 & 2 & 0.00 & 0.00 & 1.33 & 159.00 & 0.00 & 0.00 & 0.00 & 0.00 & 0.00 & 0.00 & 0.00 & 0.00 \\
\hline 6 & 2 & 0.02 & 6.00 & 2.07 & 292.00 & 0.00 & 0.00 & 0.00 & 0.00 & 0.00 & 0.00 & 0.00 & 0.00 \\
\hline 6 & 2 & 0.00 & 0.00 & 1.76 & 253.00 & 0.00 & 0.00 & 0.00 & 0.00 & 0.00 & 0.00 & 0.00 & 0.00 \\
\hline 6 & 2 & 0.00 & 0.00 & 1.90 & 274.00 & 0.00 & 0.00 & 0.00 & 0.00 & 0.00 & 0.00 & 0.00 & 0.00 \\
\hline 7 & 2 & 0.02 & 6.00 & 2.07 & 292.00 & 0.00 & 0.00 & 0.00 & 0.00 & 0.00 & 0.00 & 0.00 & 0.00 \\
\hline 7 & 2 & 0.00 & 0.00 & 1.76 & 253.00 & 0.00 & 0.00 & 0.00 & 0.00 & 0.00 & 0.00 & 0.00 & 0.00 \\
\hline 7 & 2 & 0.00 & 0.00 & 1.90 & 274.00 & 0.00 & 0.00 & 0.00 & 0.00 & 0.00 & 0.00 & 0.00 & 0.00 \\
\hline 1 & 3 & 0.00 & 0.00 & 1.35 & 191.00 & 0.00 & 0.00 & 0.00 & 0.00 & 0.00 & 0.00 & 0.00 & 0.00 \\
\hline 1 & 3 & 0.00 & 0.00 & 1.07 & 189.00 & 0.00 & 0.00 & 0.00 & 0.00 & 0.00 & 0.00 & 0.04 & 15.00 \\
\hline 1 & 3 & 0.00 & 0.00 & 1.12 & 192.00 & 0.00 & 0.00 & 0.00 & 0.00 & 0.00 & 0.00 & 0.00 & 0.00 \\
\hline 2 & 3 & 0.00 & 0.00 & 1.82 & 211.00 & 0.00 & 0.00 & 0.00 & 0.00 & 0.00 & 0.00 & 0.00 & 0.00 \\
\hline 2 & 3 & 0.00 & 0.00 & 1.97 & 266.00 & 0.00 & 0.00 & 0.00 & 0.00 & 0.00 & 0.00 & 0.00 & 0.00 \\
\hline 2 & 3 & 0.23 & 23.00 & 0.81 & 176.00 & 0.00 & 0.00 & 0.00 & 0.00 & 0.00 & 0.00 & 0.08 & 38.00 \\
\hline 3 & 3 & 0.00 & 0.00 & 1.72 & 227.00 & 0.00 & 0.00 & 0.00 & 0.00 & 0.00 & 0.00 & 0.00 & 0.00 \\
\hline 3 & 3 & 0.01 & 5.00 & 0.73 & 161.00 & 0.00 & 0.00 & 0.00 & 0.00 & 0.00 & 0.00 & 0.15 & 67.00 \\
\hline 3 & 3 & 0.00 & 0.00 & 1.15 & 219.00 & 0.04 & 5.00 & 0.00 & 0.00 & 0.00 & 0.00 & 0.23 & 95.00 \\
\hline 4 & 3 & 0.00 & 0.00 & 1.59 & 198.00 & 0.00 & 0.00 & 0.00 & 0.00 & 0.00 & 0.00 & 0.00 & 0.00 \\
\hline 4 & 3 & 0.00 & 0.00 & 2.97 & 323.00 & 0.00 & 0.00 & 0.00 & 0.00 & 0.00 & 0.00 & 0.00 & 0.00 \\
\hline 4 & 3 & 0.00 & 0.00 & 2.29 & 223.00 & 0.00 & 0.00 & 0.00 & 0.00 & 0.00 & 0.00 & 0.00 & 0.00 \\
\hline 5 & 3 & 0.00 & 0.00 & 2.23 & 289.00 & 0.00 & 0.00 & 0.00 & 0.00 & 0.00 & 0.00 & 0.00 & 0.00 \\
\hline 5 & 3 & 0.00 & 0.00 & 1.45 & 211.00 & 0.00 & 0.00 & 0.00 & 0.00 & 0.00 & 0.00 & 0.00 & 0.00 \\
\hline 5 & 3 & 0.00 & 0.00 & 1.71 & 251.00 & 0.00 & 0.00 & 0.00 & 0.00 & 0.00 & 0.00 & 0.00 & 0.00 \\
\hline
\end{tabular}




\begin{tabular}{|c|c|c|c|c|c|c|c|c|c|c|c|c|c|}
\hline 6 & 3 & 0.00 & 0.00 & 1.33 & 205.00 & 0.00 & 0.00 & 0.00 & 0.00 & 0.00 & 0.00 & 0.00 & 0.00 \\
\hline 6 & 3 & 0.00 & 0.00 & 1.04 & 187.00 & 0.00 & 0.00 & 0.00 & 0.00 & 0.00 & 0.00 & 0.04 & 16.00 \\
\hline 6 & 3 & 0.00 & 0.00 & 2.12 & 210.00 & 0.00 & 0.00 & 0.00 & 0.00 & 0.00 & 0.00 & 0.00 & 0.00 \\
\hline 7 & 3 & 0.00 & 0.00 & 1.33 & 205.00 & 0.00 & 0.00 & 0.00 & 0.00 & 0.00 & 0.00 & 0.00 & 0.00 \\
\hline 7 & 3 & 0.00 & 0.00 & 1.04 & 187.00 & 0.00 & 0.00 & 0.00 & 0.00 & 0.00 & 0.00 & 0.04 & 16.00 \\
\hline 7 & 3 & 0.00 & 0.00 & 2.12 & 210.00 & 0.00 & 0.00 & 0.00 & 0.00 & 0.00 & 0.00 & 0.00 & 0.00 \\
\hline 1 & 4 & 0.00 & 0.00 & 0.53 & 109.00 & 0.00 & 0.00 & 0.00 & 0.00 & 0.00 & 0.00 & 0.00 & 0.00 \\
\hline 1 & 4 & 0.00 & 0.00 & 0.65 & 105.00 & 0.00 & 0.00 & 0.00 & 0.00 & 0.00 & 0.00 & 0.00 & 0.00 \\
\hline 1 & 4 & 0.00 & 0.00 & 0.69 & 117.00 & 0.00 & 0.00 & 0.00 & 0.00 & 0.00 & 0.00 & 0.00 & 0.00 \\
\hline 2 & 4 & 0.00 & 0.00 & 0.65 & 121.00 & 0.00 & 0.00 & 0.00 & 0.00 & 0.00 & 0.00 & 0.00 & 0.00 \\
\hline 2 & 4 & 0.00 & 0.00 & 0.72 & 138.00 & 0.00 & 0.00 & 0.00 & 0.00 & 0.00 & 0.00 & 0.00 & 0.00 \\
\hline 2 & 4 & 0.00 & 0.00 & 0.78 & 133.00 & 0.00 & 0.00 & 0.00 & 0.00 & 0.00 & 0.00 & 0.00 & 0.00 \\
\hline 3 & 4 & 0.00 & 0.00 & 0.04 & 7.00 & 0.00 & 0.00 & 0.00 & 0.00 & 0.03 & 5.00 & 0.00 & 0.00 \\
\hline 3 & 4 & 0.00 & 0.00 & 0.80 & 125.00 & 0.00 & 0.00 & 0.00 & 0.00 & 0.00 & 0.00 & 0.00 & 0.00 \\
\hline 3 & 4 & 0.00 & 0.00 & 0.58 & 110.00 & 0.00 & 0.00 & 0.00 & 0.00 & 0.00 & 0.00 & 0.00 & 0.00 \\
\hline 4 & 4 & 0.00 & 0.00 & 0.88 & 116.00 & 0.00 & 0.00 & 0.00 & 0.00 & 0.00 & 0.00 & 0.00 & 0.00 \\
\hline 4 & 4 & 0.00 & 0.00 & 0.88 & 127.00 & 0.00 & 0.00 & 0.00 & 0.00 & 0.00 & 0.00 & 0.00 & 0.00 \\
\hline 4 & 4 & 0.00 & 0.00 & 0.69 & 92.00 & 0.00 & 0.00 & 0.00 & 0.00 & 0.00 & 0.00 & 0.00 & 0.00 \\
\hline 5 & 4 & 0.00 & 0.00 & 0.43 & 70.00 & 0.00 & 0.00 & 0.04 & 6.00 & 0.03 & 5.00 & 0.00 & 0.00 \\
\hline 5 & 4 & 0.00 & 0.00 & 0.61 & 100.00 & 0.00 & 0.00 & 0.00 & 0.00 & 0.00 & 0.00 & 0.00 & 0.00 \\
\hline 5 & 4 & 0.00 & 0.00 & 0.66 & 104.00 & 0.00 & 0.00 & 0.00 & 0.00 & 0.00 & 0.00 & 0.00 & 0.00 \\
\hline 6 & 4 & 0.00 & 0.00 & 0.59 & 118.00 & 0.00 & 0.00 & 0.00 & 0.00 & 0.00 & 0.00 & 0.00 & 0.00 \\
\hline 6 & 4 & 0.00 & 0.00 & 0.84 & 128.00 & 0.00 & 0.00 & 0.00 & 0.00 & 0.00 & 0.00 & 0.00 & 0.00 \\
\hline 6 & 4 & 0.00 & 0.00 & 0.68 & 86.00 & 0.00 & 0.00 & 0.00 & 0.00 & 0.00 & 0.00 & 0.00 & 0.00 \\
\hline 7 & 4 & 0.00 & 0.00 & 0.59 & 118.00 & 0.00 & 0.00 & 0.00 & 0.00 & 0.00 & 0.00 & 0.00 & 0.00 \\
\hline 7 & 4 & 0.00 & 0.00 & 0.84 & 128.00 & 0.00 & 0.00 & 0.00 & 0.00 & 0.00 & 0.00 & 0.00 & 0.00 \\
\hline 7 & 4 & 0.00 & 0.00 & 0.68 & 86.00 & 0.00 & 0.00 & 0.00 & 0.00 & 0.00 & 0.00 & 0.00 & 0.00 \\
\hline
\end{tabular}


Table S6. Example of matrix input of variables for building the PCA model (Part 3).

\begin{tabular}{|c|c|c|c|c|c|c|c|c|c|c|c|c|c|}
\hline ay & $\begin{array}{c}\text { Crude } \\
\text { oil }\end{array}$ & $\begin{array}{l}\% \mathrm{~S} \\
\text { Class }\end{array}$ & $\begin{array}{l}\text { Total } N^{\circ} \text { of } \\
\mathrm{S} \\
\text { compounds }\end{array}$ & $\begin{array}{c}\text { DBE } \\
\mathrm{N}[\mathrm{H}] 3\end{array}$ & $\begin{array}{c}\mathrm{DBE} \\
\mathrm{N}[\mathrm{H}] 4\end{array}$ & $\begin{array}{c}\mathrm{DBE} \\
\mathrm{N}[\mathrm{H}] 5\end{array}$ & $\begin{array}{c}\mathrm{DBE} \\
\mathrm{N}[\mathrm{H}] 6\end{array}$ & $\begin{array}{c}\text { DBE } \\
\mathrm{N}[\mathrm{H}] 7\end{array}$ & $\begin{array}{c}\mathrm{DBE} \\
\mathrm{N}[\mathrm{H}] 8\end{array}$ & $\begin{array}{c}\mathrm{DBE} \\
\mathrm{N}[\mathrm{H}] 9\end{array}$ & $\begin{array}{c}\mathrm{DBE} \\
\mathrm{N}[\mathrm{H}] \\
10\end{array}$ & $\begin{array}{c}\text { DBE } \\
N[H] 11\end{array}$ & $\begin{array}{c}\text { DBE } \\
\mathrm{N}[\mathrm{H}] \\
12\end{array}$ \\
\hline 1 & 1 & 0.00 & 0.00 & 0.1150 & 1.3350 & 3.1780 & 5.6630 & 6.9900 & 7.9120 & 8.7200 & 8.7200 & 7.9350 & 7.2310 \\
\hline 1 & 1 & 0.00 & 0.00 & 0.1580 & 1.1110 & 2.8770 & 4.4660 & 5.2750 & 6.0430 & 7.2640 & 6.9780 & 7.1450 & 6.4760 \\
\hline 1 & 1 & 0.00 & 0.00 & 0.1740 & 1.3010 & 2.9720 & 4.6380 & 5.5450 & 6.6070 & 7.3980 & 7.2030 & 7.1880 & 6.6240 \\
\hline 2 & 1 & 0.00 & 0.00 & 0.1170 & 0.9680 & 2.4650 & 4.0750 & 5.0600 & 6.0580 & 6.7630 & 6.7290 & 6.7400 & 6.0210 \\
\hline 2 & 1 & 0.00 & 0.00 & 0.0720 & 0.8570 & 2.0280 & 3.5940 & 4.3950 & 5.3430 & 6.1770 & 6.2920 & 6.2900 & 5.7160 \\
\hline 2 & 1 & 0.00 & 0.00 & 0.1510 & 1.0860 & 2.6680 & 4.3880 & 5.3620 & 6.2340 & 6.7830 & 6.8420 & 6.9160 & 6.3330 \\
\hline 3 & 1 & 0.00 & 0.00 & 0.1510 & 1.1840 & 2.7630 & 4.3940 & 5.4540 & 6.5610 & 7.1590 & 7.1800 & 7.2260 & 6.6550 \\
\hline 3 & 1 & 0.00 & 0.00 & 0.1580 & 1.1810 & 2.7360 & 4.4050 & 5.4870 & 6.5650 & 6.9150 & 7.2010 & 7.1750 & 6.5470 \\
\hline 3 & 1 & 0.00 & 0.00 & 0.1450 & 1.1490 & 2.7750 & 4.3640 & 5.4670 & 6.4230 & 7.1360 & 7.1840 & 7.1520 & 6.6860 \\
\hline 4 & 1 & 0.00 & 0.00 & 0.2410 & 1.6700 & 3.8370 & 6.2820 & 7.6950 & 8.5870 & 8.9880 & 8.6580 & 7.4 & 6.6680 \\
\hline 4 & 1 & 0.00 & 0.00 & 0.2150 & 1.5560 & 3.6880 & 6.0920 & 7.6890 & 8.4560 & 9.1510 & 8.9870 & 7.9 & 6.9030 \\
\hline 4 & 1 & 0.00 & 0.00 & 0.2100 & 1.6060 & 3.8430 & 6.1870 & 7.4140 & 8.5090 & 9.0910 & 8.8800 & 8.1410 & 7.2430 \\
\hline 5 & 1 & 0.00 & 0.00 & 0.2100 & 1.2590 & 3.1100 & 4.9220 & 6.2600 & 7.4350 & 8.0870 & 7.5560 & 7.6 & 7.0480 \\
\hline 5 & 1 & 0.00 & 0.00 & 0.1250 & 1.0750 & 2.5360 & 4.1340 & 5.1730 & & 7.1620 & 7.0760 & & 6.2770 \\
\hline 5 & 1 & 0.00 & 0.00 & 0.1840 & 1.1340 & 2.5630 & 4.7440 & 5.8320 & 6.5050 & 6.7470 & 7.8990 & 7.0710 & 6.7080 \\
\hline 6 & 1 & 0.00 & 0.00 & 0.1620 & 1.6530 & 3.9240 & 6.3650 & 7.4870 & 8.4230 & 8.6650 & 8.3960 & 7.5 & 6.8150 \\
\hline 6 & 1 & 0.00 & 0.00 & 0.3270 & 2.2660 & 4.7530 & 7.0510 & 8.5930 & & 8.9000 & 8.2540 & & 6.8900 \\
\hline 6 & 1 & 0.00 & 0.00 & 0.1520 & 1.1930 & 2.8160 & 4.4510 & 5.5420 & 6.3260 & 7.2280 & 7.0820 & 6.9020 & 6.7070 \\
\hline 7 & 1 & 0.00 & 0.00 & 0.1620 & 1.6530 & 3.9240 & 6.3650 & 7.4870 & 8.4230 & 8.6650 & 8.3960 & 7.5590 & 6.8150 \\
\hline 7 & 1 & 0.00 & 0.00 & 0.3270 & 2.2660 & 4.7530 & 7.0510 & 8.5930 & 8.9070 & 8.9000 & 8.2540 & 7.8370 & 6.8900 \\
\hline 7 & 1 & 0.00 & 0.00 & 0.1520 & 1.1930 & 2.8160 & 4.4510 & 5.5420 & 6.3260 & 7.2280 & 7.0820 & 6.9020 & 6.7070 \\
\hline 1 & 2 & 0.00 & 0.00 & 0.2830 & 1.6410 & 3.4170 & 5.1350 & 6.5490 & 7.2720 & 7.6600 & 7.7720 & 7.4950 & 7.0050 \\
\hline 1 & 2 & 0.00 & 0.00 & 0.2880 & 2.3660 & 4.8310 & 6.8160 & 7.7700 & 8.7660 & 9.0100 & 8.5990 & 8.0260 & 7.1360 \\
\hline 1 & 2 & 0.00 & 0.00 & 0.2470 & 1.9650 & 4.2680 & 5.9890 & 7.6700 & 8.1960 & 8.7030 & 8.0720 & 7.7340 & 6.9340 \\
\hline 2 & 2 & 0.00 & 0.00 & 0.3770 & 1.6900 & 3.5550 & 5.1250 & 6.7540 & 7.4990 & 7.6890 & 7.9320 & 7.6780 & 6.9230 \\
\hline 2 & 2 & 0.00 & 0.00 & 0.3590 & 1.7640 & 3.6810 & 5.4540 & 6.8850 & 7.6360 & 7.7040 & 7.8410 & 7.5700 & 6.9040 \\
\hline
\end{tabular}




\begin{tabular}{|c|c|c|c|c|c|c|c|c|c|c|c|c|c|}
\hline 2 & 2 & 0.00 & 0.00 & 0.2610 & 2.2040 & 4.5010 & 6.5650 & 8.0260 & 8.8850 & 8.7510 & 8.0450 & 7.4780 & 6.9630 \\
\hline 3 & 2 & 0.00 & 0.00 & 0.5730 & 1.4870 & 2.8940 & 4.6040 & 5.7930 & 6.6520 & 7.0570 & 6.8150 & 6.8460 & 6.5790 \\
\hline 3 & 2 & 0.00 & 0.00 & 0.4890 & 1.4540 & 3.0940 & 4.8540 & 6.2180 & 6.6310 & 7.0850 & 6.9660 & 6.9060 & 6.7210 \\
\hline 3 & 2 & 0.00 & 0.00 & 0.4900 & 1.5670 & 3.0580 & 4.7960 & 6.2210 & 6.7620 & 7.2210 & 6.9710 & 7.0660 & 6.5750 \\
\hline 4 & 2 & 0.00 & 0.00 & 0.2550 & 2.4890 & 5.5220 & 7.8000 & 9.0030 & 9.5420 & 9.4540 & 8.3590 & 6.9490 & 6.8020 \\
\hline 4 & 2 & 0.00 & 0.00 & 0.2750 & 2.4880 & 5.0470 & 7.1650 & 8.4940 & 9.6700 & 9.6390 & 8.5600 & 8.1470 & 7.0100 \\
\hline 4 & 2 & 0.00 & 0.00 & 0.2830 & 2.2740 & 4.9290 & 6.9980 & 8.9700 & 9.5220 & 9.2950 & 8.4360 & 8.1160 & 7.0700 \\
\hline 5 & 2 & 0.00 & 0.00 & 0.2140 & 2.0030 & 3.9720 & 6.5450 & 8.4910 & 8.2240 & 9.3460 & 8.6130 & 7.9880 & 7.1270 \\
\hline 5 & 2 & 0.00 & 0.00 & 0.2590 & 1.9710 & 4.1040 & 6.0040 & 7.4970 & 8.1680 & 8.1860 & 8.0930 & 7.7940 & 7.0030 \\
\hline 5 & 2 & 0.00 & 0.00 & 0.1900 & 2.0010 & 4.2880 & 6.7320 & 7.5170 & 8.5020 & 8.2900 & 8.4140 & 7.4540 & 7.0320 \\
\hline 6 & 2 & 0.00 & 0.00 & 0.3740 & 1.7110 & 3.4290 & 5.2770 & 6.8070 & 7.7370 & 7.7860 & 7.9150 & 7.5250 & 6.7830 \\
\hline 6 & 2 & 0.00 & 0.00 & 0.3770 & 1.6900 & 3.5550 & 5.1250 & 6.7540 & 7.4990 & 7.6890 & 7.9320 & 7.6780 & 6.9230 \\
\hline 6 & 2 & 0.00 & 0.00 & 0.3980 & 1.6880 & 3.5460 & 5.1090 & 6.4960 & 7.6760 & 8.1290 & 7.7080 & 7.4410 & 6.8310 \\
\hline 7 & 2 & 0.00 & 0.00 & 0.3740 & 1.7110 & 3.4290 & 5.2770 & 6.8070 & 7.7370 & 7.7860 & 7.9150 & 7.5250 & 6.7830 \\
\hline 7 & 2 & 0.00 & 0.00 & 0.3770 & 1.6900 & 3.5550 & 5.1250 & 6.7540 & 7.4990 & 7.6890 & 7.9320 & 7.6780 & 6.9230 \\
\hline 7 & 2 & 0.00 & 0.00 & 0.3970 & 1.6860 & 3.5440 & 5.1060 & 6.4910 & 7.6710 & 8.1230 & 7.7020 & 7.4360 & 6.8470 \\
\hline 1 & 3 & 0.00 & 0.00 & 0.4160 & 2.7920 & 5.1900 & 7.3660 & 9.1070 & 9.3670 & 8.3770 & 8.0920 & 7.9300 & 6.7380 \\
\hline 1 & 3 & 0.00 & 0.00 & 0.4290 & 2.5650 & 5.1940 & 7.2320 & 8.4970 & 8.9840 & 8.7780 & 8.2540 & 7.7960 & 6.8830 \\
\hline 1 & 3 & 0.00 & 0.00 & 0.3130 & 2.3280 & 4.7430 & 6.7600 & 8.0970 & 8.6380 & 8.6580 & 8.2290 & 7.7470 & 7.0370 \\
\hline 2 & 3 & 0.00 & 0.00 & 0.3930 & 2.9590 & 5.9410 & 8.4040 & 9.9690 & 10.0690 & 9.4020 & 8.5370 & 7.2060 & 6.2350 \\
\hline 2 & 3 & 0.00 & 0.00 & 0.3750 & 2.7780 & 5.5520 & 7.6330 & 9.3170 & 9.7450 & 9.0860 & 8.4550 & 7.3470 & 6.3970 \\
\hline 2 & 3 & 0.00 & 0.00 & 0.4300 & 2.1820 & 4.2720 & 6.0940 & 7.5530 & 8.0410 & 8.3920 & 7.9680 & 7.4960 & 6.9390 \\
\hline 3 & 3 & 0.00 & 0.00 & 0.3500 & 2.1840 & 4.4400 & 6.3140 & 8.0910 & 8.2610 & 8.7420 & 8.0980 & 7.6280 & 6.8150 \\
\hline 3 & 3 & 0.00 & 0.00 & 0.4450 & 2.1360 & 4.2310 & 5.9910 & 7.2850 & 8.1880 & 8.1600 & 8.0030 & 7.6330 & 6.8140 \\
\hline 3 & 3 & 0.00 & 0.00 & 0.4260 & 2.1840 & 4.2350 & 6.0070 & 7.2710 & 8.1290 & 8.2250 & 8.0950 & 7.8560 & 7.0100 \\
\hline 4 & 3 & 0.04 & 5.00 & 0.3870 & 2.7900 & 5.7950 & 7.9100 & 9.6080 & 9.8260 & 9.8340 & 8.3730 & 7.9730 & 6.9300 \\
\hline 4 & 3 & 0.00 & 0.00 & 0.3210 & 2.8350 & 5.8630 & 8.8250 & 9.5360 & 10.3290 & 10.6330 & 8.3970 & 7.4440 & 6.6700 \\
\hline 4 & 3 & 0.00 & 0.00 & 0.3530 & 2.8010 & 6.0910 & 8.8400 & 10.0200 & 10.1230 & 10.1410 & 8.9680 & 7.2620 & 6.4310 \\
\hline 5 & 3 & 0.00 & 0.00 & 0.2990 & 2.3000 & 4.5190 & 6.7000 & 8.5660 & 9.0240 & 9.1370 & 8.8650 & 7.5910 & 6.7110 \\
\hline 5 & 3 & 0.00 & 0.00 & 0.3270 & 2.2660 & 4.7530 & 7.0510 & 8.5930 & 8.9070 & 8.9000 & 8.2540 & 7.8370 & 6.8900 \\
\hline 5 & 3 & 0.00 & 0.00 & 0.3510 & 2.5190 & 4.9430 & 6.8990 & 8.4790 & 9.0060 & 8.5620 & 8.1850 & 7.6510 & 6.7540 \\
\hline 6 & 3 & 0.00 & 0.00 & 0.3140 & 2.5380 & 5.0950 & 6.7740 & 8.4930 & 8.9630 & 8.7410 & 8.1900 & 7.6410 & 6.8840 \\
\hline
\end{tabular}




\begin{tabular}{|c|c|c|c|c|c|c|c|c|c|c|c|c|c|}
\hline 6 & 3 & 0.00 & 0.00 & 0.4240 & 2.6500 & 5.4120 & 7.1340 & 8.5070 & 8.9960 & 8.8030 & 8.2540 & 7.9620 & 6.8750 \\
\hline 6 & 3 & 0.00 & 0.00 & 0.3720 & 2.7340 & 5.8830 & 8.6610 & 8.8730 & 10.5080 & 9.7930 & 9.0920 & 7.5150 & 6.3670 \\
\hline 7 & 3 & 0.00 & 0.00 & 0.3140 & 2.5380 & 5.0950 & 6.7740 & 8.4930 & 8.9630 & 8.7410 & 8.1900 & 7.6410 & 6.8840 \\
\hline 7 & 3 & 0.00 & 0.00 & 0.4240 & 2.6500 & 5.4120 & 7.1340 & 8.5070 & 8.9960 & 8.8030 & 8.2540 & 7.9620 & 6.8750 \\
\hline 7 & 3 & 0.00 & 0.00 & 0.3720 & 2.7340 & 5.8830 & 8.6610 & 8.8730 & 10.5080 & 9.7930 & 9.0920 & 7.5150 & 6.3670 \\
\hline 1 & 4 & 0.00 & 0.00 & 0.1130 & 1.5170 & 4.2320 & 6.7190 & 7.9760 & 9.0420 & 9.4150 & 8.9410 & 9.0100 & 7.5640 \\
\hline 1 & 4 & 0.00 & 0.00 & 0.1700 & 1.7730 & 5.1180 & 8.4080 & 9.9430 & 10.4280 & 10.9390 & 9.4720 & 9.2880 & 7.4720 \\
\hline 1 & 4 & 0.00 & 0.00 & 0.1630 & 1.7140 & 4.7780 & 7.4420 & 9.0180 & 9.8340 & 10.3370 & 9.6180 & 9.4570 & 7.4980 \\
\hline 2 & 4 & 0.00 & 0.00 & 0.1340 & 1.4220 & 3.4810 & 6.5430 & 7.9920 & 9.2620 & 9.1870 & 8.9960 & 8.9530 & 7.9810 \\
\hline 2 & 4 & 0.00 & 0.00 & 0.1140 & 1.4340 & 3.9790 & 6.3190 & 7.8090 & 8.7200 & 9.1860 & 9.1490 & 9.3660 & 7.8400 \\
\hline 2 & 4 & 0.00 & 0.00 & 0.1250 & 1.2290 & 3.3150 & 5.3810 & 6.8090 & 7.4560 & 8.3730 & 8.3330 & 8.4510 & 7.8680 \\
\hline 3 & 4 & 0.00 & 0.00 & 0.0000 & 0.7320 & 2.3270 & 3.9660 & 5.2080 & 6.3470 & 7.0440 & 7.2630 & 7.2530 & 6.6910 \\
\hline 3 & 4 & 0.00 & 0.00 & 0.0880 & 1.0870 & 3.1490 & 5.4580 & 6.8170 & 7.8960 & 8.5140 & 8.8780 & 8.6330 & 7.6230 \\
\hline 3 & 4 & 0.00 & 0.00 & 0.0810 & 0.9810 & 2.7040 & 4.7690 & 6.1470 & 6.8970 & 8.0910 & 8.2920 & 7.7150 & 7.1660 \\
\hline 4 & 4 & 0.00 & 0.00 & 0.1050 & 1.4930 & 4.2150 & 6.7810 & 8.5110 & 9.5910 & 10.5100 & 9.7220 & 10.1160 & 8.1560 \\
\hline 4 & 4 & 0.00 & 0.00 & 0.1220 & 1.4800 & 4.2410 & 6.9650 & 8.4040 & 9.7420 & 10.5010 & 9.6380 & 9.9530 & 8.2520 \\
\hline 4 & 4 & 0.00 & 0.00 & 0.1120 & 1.3420 & 3.9650 & 6.4460 & 7.9920 & 9.7570 & 9.5330 & 9.9060 & 9.6170 & 8.0480 \\
\hline 5 & 4 & 0.00 & 0.00 & 0.0630 & 1.0170 & 2.9510 & 5.2260 & 6.6480 & 7.3780 & 8.5400 & 8.4500 & 7.8530 & 6.8240 \\
\hline 5 & 4 & 0.00 & 0.00 & 0.0850 & 1.1500 & 3.1600 & 5.5620 & 7.1280 & 7.7800 & 8.7150 & 8.8490 & 8.0440 & 6.9450 \\
\hline 5 & 4 & 0.00 & 0.00 & 0.0960 & 1.0730 & 3.2590 & 6.1980 & 6.9690 & 7.4740 & 8.7640 & 8.7110 & 8.1240 & 7.3860 \\
\hline 6 & 4 & 0.00 & 0.00 & 0.1440 & 1.5320 & 4.2540 & 6.8710 & 7.5420 & 9.1370 & 9.6540 & 8.6140 & 9.1090 & 7.7550 \\
\hline 6 & 4 & 0.00 & 0.00 & 0.0890 & 1.1190 & 2.9280 & 5.0980 & 6.4710 & 7.7290 & 8.4150 & 8.8270 & 8.8610 & 7.8100 \\
\hline 6 & 4 & 0.00 & 0.00 & 0.1190 & 1.2840 & 3.7380 & 5.9800 & 7.9240 & 9.7250 & 9.4460 & 9.9830 & 9.6710 & 8.4520 \\
\hline 7 & 4 & 0.00 & 0.00 & 0.1440 & 1.5320 & 4.2540 & 6.8710 & 7.5420 & 9.1370 & 9.6540 & 8.6140 & 9.1090 & 7.7550 \\
\hline 7 & 4 & 0.00 & 0.00 & 0.0890 & 1.1190 & 2.9280 & 5.0980 & 6.4710 & 7.7290 & 8.4150 & 8.8270 & 8.8610 & 7.8100 \\
\hline 7 & 4 & 0.00 & 0.00 & 0.1190 & 1.2840 & 3.7380 & 5.9800 & 7.9240 & 9.7250 & 9.4460 & 9.9830 & 9.6710 & 8.4520 \\
\hline
\end{tabular}


Table S7. Example of matrix input of variables for building the PCA model (Part 4).

\begin{tabular}{|c|c|c|c|c|c|c|c|c|c|c|c|c|c|}
\hline Day & $\begin{array}{c}\text { Crude } \\
\text { oil }\end{array}$ & $\begin{array}{c}\mathrm{DBE} \\
\mathrm{N}[\mathrm{H}] \\
13\end{array}$ & $\begin{array}{c}\mathrm{DBE} \\
\mathrm{N}[\mathrm{H}] \\
14\end{array}$ & $\begin{array}{c}\mathrm{DBE} \\
\mathrm{N}[\mathrm{H}] \\
15\end{array}$ & $\begin{array}{c}\mathrm{DBE} \\
\mathrm{N}[\mathrm{H}] \\
16\end{array}$ & $\begin{array}{c}\mathrm{DBE} \\
\mathrm{N}[\mathrm{H}] \\
17\end{array}$ & $\begin{array}{c}\mathrm{DBE} \\
\mathrm{N}[\mathrm{H}] \\
18\end{array}$ & $\begin{array}{c}\text { DBE } \\
\mathrm{N}[\mathrm{H}] \\
19\end{array}$ & $\begin{array}{c}\mathrm{DBE} \\
\mathrm{N}[\mathrm{H}] \\
20\end{array}$ & $\begin{array}{c}\mathrm{DBE} \\
\mathrm{N}[\mathrm{H}] \\
21\end{array}$ & $\begin{array}{c}\mathrm{DBE} \\
\mathrm{N}[\mathrm{H}] \\
22\end{array}$ & $\begin{array}{c}\mathrm{DBE} \\
\mathrm{N}[\mathrm{H}] \\
23\end{array}$ & $\begin{array}{c}\mathrm{DBE} \\
\mathrm{N}[\mathrm{H}] \\
24\end{array}$ \\
\hline 1 & 1 & 6.2230 & 5.5670 & 4.7800 & 3.8040 & 3.2800 & 2.5720 & 2.1770 & 1.7500 & 1.4370 & 1.1840 & 0.9580 & 0.6950 \\
\hline 1 & 1 & 5.5890 & 5.4080 & 4.8470 & 4.1670 & 3.3380 & 2.9730 & 2.4460 & 2.1330 & 1.8120 & 1.4490 & 1.1930 & 0.8490 \\
\hline 1 & 1 & 5.8870 & 5.2440 & 4.5370 & 3.9400 & 3.3860 & 2.8570 & 2.3870 & 2.0490 & 1.7070 & 1.3500 & 1.1440 & 0.9720 \\
\hline 2 & 1 & 5.4710 & 4.9350 & 4.2370 & 3.6410 & 3.0490 & 2.5810 & 2.2850 & 1.8740 & 1.5780 & 1.3740 & 1.0610 & 0.9240 \\
\hline 2 & 1 & 5.2110 & 4.6570 & 4.1660 & 3.6940 & 3.1920 & 2.6220 & 2.3700 & 1.7880 & 1.5340 & 1.3800 & 1.1380 & 0.8670 \\
\hline 2 & 1 & 5.7490 & 5.2630 & 4.5100 & 3.7890 & 3.3080 & 2.9550 & 2.4730 & 2.0910 & 1.8770 & 1.4920 & 1.2820 & 1.0090 \\
\hline 3 & 1 & 5.9060 & 5.3060 & 4.5890 & 3.9780 & 3.3090 & 2.7310 & 2.4080 & 1.9820 & 1.6710 & 1.3190 & 1.1560 & 0.9390 \\
\hline 3 & 1 & 5.7500 & 5.2350 & 4.7000 & 3.9310 & 3.2880 & 2.8760 & 2.4320 & 2.0090 & 1.7030 & 1.4120 & 1.1810 & 0.9480 \\
\hline 3 & 1 & 5.9160 & 5.2940 & 4.6730 & 3.9430 & 3.3220 & 2.8890 & 2.4190 & 2.0010 & 1.6670 & 1.3580 & 1.1860 & 0.9360 \\
\hline 4 & 1 & 5.6160 & 4.8140 & 4.1030 & 3.3000 & 2.8640 & 2.2680 & 1.9060 & 1.4920 & 1.2020 & 0.9700 & 0.7930 & 0.6060 \\
\hline 4 & 1 & 5.8670 & 5.2160 & 4.1620 & 3.3290 & 2.7160 & 2.2370 & 1.7410 & 1.3360 & 1.0970 & 0.8570 & 0.6720 & 0.5060 \\
\hline 4 & 1 & 6.1780 & 5.5170 & 4.4720 & 3.7230 & 2.8890 & 2.2980 & 1.8830 & 1.4480 & 1.1720 & 0.8240 & 0.6980 & 0.5110 \\
\hline 5 & 1 & 5.9590 & 5.1740 & 4.2690 & 3.6540 & 3.1160 & 2.8530 & 2.2990 & 1.8040 & 1.5250 & 1.2180 & 1.1000 & 0.8570 \\
\hline 5 & 1 & 5.5590 & 4.9760 & 4.3190 & 3.5870 & 2.9970 & 2.5810 & 2.1000 & 1.5820 & 1.3670 & 1.1240 & 0.9510 & 0.7670 \\
\hline 5 & 1 & 4.7740 & 4.6110 & 4.4010 & 3.5000 & 3.2890 & 2.6500 & 2.3730 & 1.9000 & 1.7040 & 1.4530 & 1.1130 & 0.9490 \\
\hline 6 & 1 & 5.8980 & 4.8110 & 4.0930 & 3.3930 & 2.9250 & 2.3180 & 1.8590 & 1.5100 & 1.2050 & 0.9320 & 0.7240 & 0.5810 \\
\hline 6 & 1 & 5.7890 & 4.8480 & 3.7860 & 3.1330 & 2.6100 & 2.0650 & 1.5120 & 1.1310 & 0.9480 & 0.7100 & 0.5100 & 0.4260 \\
\hline 6 & 1 & 5.8110 & 5.1660 & 4.5130 & 3.9260 & 3.3220 & 2.8030 & 2.4330 & 2.0260 & 1.7130 & 1.3920 & 1.1000 & 0.8970 \\
\hline 7 & 1 & 5.8980 & 4.8110 & 4.0930 & 3.3930 & 2.9250 & 2.3180 & 1.8590 & 1.5100 & 1.2050 & 0.9320 & 0.7240 & 0.5810 \\
\hline 7 & 1 & 5.7890 & 4.8480 & 3.7860 & 3.1330 & 2.6100 & 2.0650 & 1.5120 & 1.1310 & 0.9480 & 0.7100 & 0.5100 & 0.4260 \\
\hline 7 & 1 & 5.8110 & 5.1660 & 4.5130 & 3.9260 & 3.3220 & 2.8030 & 2.4330 & 2.0260 & 1.7130 & 1.3920 & 1.1000 & 0.8970 \\
\hline 1 & 2 & 6.0660 & 5.5440 & 4.9100 & 4.1270 & 3.5010 & 2.8990 & 2.3780 & 1.8040 & 1.5040 & 1.2150 & 0.8640 & 0.7540 \\
\hline 1 & 2 & 6.0910 & 4.8010 & 4.0460 & 3.2460 & 2.7590 & 2.0700 & 1.6360 & 1.2060 & 0.9230 & 0.6490 & 0.5100 & 0.4230 \\
\hline 1 & 2 & 5.9770 & 5.1320 & 4.3010 & 3.8490 & 3.2070 & 2.5830 & 1.9920 & 1.5990 & 1.2710 & 0.9950 & 0.7630 & 0.5750 \\
\hline 2 & 2 & 6.2080 & 5.2640 & 4.5140 & 3.6320 & 3.2050 & 2.5560 & 1.9550 & 1.6660 & 1.2690 & 1.0240 & 0.8760 & 0.6480 \\
\hline 2 & 2 & 5.9860 & 5.1460 & 4.5140 & 3.6960 & 3.0530 & 2.5160 & 1.9470 & 1.6550 & 1.2540 & 0.9770 & 0.8240 & 0.6350 \\
\hline
\end{tabular}




\begin{tabular}{|c|c|c|c|c|c|c|c|c|c|c|c|c|c|}
\hline 2 & 2 & 5.8550 & 5.1120 & 4.2420 & 3.5240 & 2.7000 & 2.2180 & 1.6910 & 1.2810 & 1.0210 & 0.7520 & 0.6100 & 0.4310 \\
\hline 3 & 2 & 5.6630 & 5.5120 & 4.7810 & 4.2080 & 3.6500 & 3.0610 & 2.6340 & 2.1160 & 1.8040 & 1.3510 & 1.1030 & 0.8830 \\
\hline 3 & 2 & 6.1310 & 5.6160 & 4.8670 & 4.0690 & 3.5260 & 3.0610 & 2.5140 & 1.9900 & 1.6050 & 1.2090 & 1.0180 & 0.8390 \\
\hline 3 & 2 & 6.0610 & 5.6120 & 4.9130 & 4.2520 & 3.6620 & 3.0910 & 2.6310 & 2.0490 & 1.7320 & 1.3190 & 1.0490 & 0.8550 \\
\hline 4 & 2 & 5.6610 & 4.6680 & 3.7170 & 2.9890 & 2.3290 & 1.7660 & 1.3630 & 1.0040 & 0.7490 & 0.5670 & 0.4260 & 0.2580 \\
\hline 4 & 2 & 5.8160 & 4.6430 & 3.6750 & 3.1290 & 2.3180 & 1.8500 & 1.3980 & 1.0940 & 0.8150 & 0.5930 & 0.4110 & 0.3070 \\
\hline 4 & 2 & 5.6550 & 4.5150 & 3.7300 & 3.0970 & 2.2220 & 1.8300 & 1.4300 & 1.0510 & 0.7930 & 0.6290 & 0.4130 & 0.3180 \\
\hline 5 & 2 & 5.9730 & 5.0390 & 4.0420 & 3.4560 & 2.7270 & 2.1200 & 1.5830 & 1.2450 & 0.9860 & 0.6740 & 0.4740 & 0.3190 \\
\hline 5 & 2 & 6.0240 & 5.1160 & 4.3320 & 3.6190 & 3.0020 & 2.4210 & 1.8570 & 1.4440 & 1.1230 & 0.9540 & 0.7050 & 0.5840 \\
\hline 5 & 2 & 6.5300 & 5.3480 & 4.3930 & 3.6750 & 3.1850 & 2.3540 & 2.0300 & 1.4850 & 1.1740 & 0.9140 & 0.6530 & 0.4580 \\
\hline 6 & 2 & 5.9430 & 5.1430 & 4.4560 & 3.6880 & 3.0870 & 2.5960 & 2.0480 & 1.6820 & 1.3310 & 1.0650 & 0.8650 & 0.6880 \\
\hline 6 & 2 & 6.2080 & 5.2640 & 4.5140 & 3.6320 & 3.2050 & 2.5560 & 1.9550 & 1.6660 & 1.2690 & 1.0240 & 0.8760 & 0.6480 \\
\hline 6 & 2 & 6.0780 & 5.2810 & 4.5250 & 3.8110 & 3.1650 & 2.5480 & 2.0020 & 1.6040 & 1.2870 & 1.0900 & 0.8510 & 0.6970 \\
\hline 7 & 2 & 5.9430 & 5.1430 & 4.4560 & 3.6880 & 3.0870 & 2.5960 & 2.0480 & 1.6820 & 1.3310 & 1.0650 & 0.8650 & 0.6880 \\
\hline 7 & 2 & 6.2080 & 5.2640 & 4.5140 & 3.6320 & 3.2050 & 2.5560 & 1.9550 & 1.6660 & 1.2690 & 1.0240 & 0.8760 & 0.6480 \\
\hline 7 & 2 & 6.0740 & 5.2780 & 4.5220 & 3.8090 & 3.1630 & 2.5460 & 2.0200 & 1.6020 & 1.2980 & 1.0850 & 0.8510 & 0.6960 \\
\hline 1 & 3 & 5.7930 & 4.9120 & 4.0170 & 3.1440 & 2.5150 & 1.9350 & 1.4620 & 1.1750 & 0.9100 & 0.6490 & 0.4190 & 0.2890 \\
\hline 1 & 3 & 5.7880 & 4.8060 & 4.1840 & 3.1310 & 2.6580 & 1.9410 & 1.5990 & 1.1020 & 0.9060 & 0.7090 & 0.5020 & 0.4000 \\
\hline 1 & 3 & 5.9680 & 5.0780 & 4.1110 & 3.4010 & 2.8180 & 2.2780 & 1.6870 & 1.3030 & 1.0500 & 0.7870 & 0.5850 & 0.4440 \\
\hline 2 & 3 & 5.1100 & 4.3630 & 3.4580 & 2.6710 & 2.0440 & 1.5470 & 1.1460 & 0.9260 & 0.6930 & 0.4970 & 0.3530 & 0.2730 \\
\hline 2 & 3 & 5.4860 & 4.5420 & 3.7940 & 2.9830 & 2.2180 & 1.8180 & 1.3620 & 0.9870 & 0.7910 & 0.5650 & 0.4070 & 0.2990 \\
\hline 2 & 3 & 6.0770 & 5.1980 & 4.5540 & 3.8130 & 3.0960 & 2.4680 & 2.0510 & 1.5440 & 1.2380 & 0.9460 & 0.6940 & 0.5230 \\
\hline 3 & 3 & 5.7970 & 5.1780 & 4.1960 & 3.4760 & 2.7660 & 2.1650 & 1.6370 & 1.2230 & 0.9770 & 0.7350 & 0.5480 & 0.3910 \\
\hline 3 & 3 & 6.0780 & 5.3310 & 4.5400 & 3.7590 & 3.1070 & 2.5550 & 2.0140 & 1.5800 & 1.2590 & 0.9660 & 0.7050 & 0.5720 \\
\hline 3 & 3 & 6.0170 & 5.2280 & 4.4200 & 3.7400 & 3.0180 & 2.3900 & 1.9590 & 1.4410 & 1.2020 & 0.9220 & 0.6850 & 0.5630 \\
\hline 4 & 3 & 5.5490 & 4.4350 & 3.5330 & 2.7210 & 2.0470 & 1.6070 & 1.1980 & 0.8510 & 0.6710 & 0.4550 & 0.2800 & 0.2230 \\
\hline 4 & 3 & 5.4920 & 4.2390 & 3.4520 & 2.5340 & 1.8490 & 1.4300 & 1.0180 & 0.7470 & 0.5710 & 0.3130 & 0.1480 & 0.1440 \\
\hline 4 & 3 & 5.2430 & 4.2890 & 3.3270 & 2.4650 & 1.8370 & 1.3720 & 0.9790 & 0.6720 & 0.5440 & 0.2960 & 0.1510 & 0.1530 \\
\hline 5 & 3 & 5.6620 & 4.8620 & 4.0140 & 3.1710 & 2.4540 & 1.8840 & 1.4250 & 1.0570 & 0.8290 & 0.5490 & 0.3720 & 0.2760 \\
\hline 5 & 3 & 5.7890 & 4.8480 & 3.7860 & 3.1330 & 2.6100 & 2.0650 & 1.5120 & 1.1310 & 0.9480 & 0.7100 & 0.5100 & 0.4260 \\
\hline 5 & 3 & 5.6090 & 4.8520 & 3.9620 & 3.1780 & 2.5960 & 2.0220 & 1.4640 & 1.1160 & 0.8520 & 0.6360 & 0.4440 & 0.3580 \\
\hline 6 & 3 & 5.6710 & 4.9040 & 4.0310 & 3.1720 & 2.6380 & 2.1140 & 1.5650 & 1.1790 & 0.8880 & 0.6620 & 0.4850 & 0.3720 \\
\hline
\end{tabular}




\begin{tabular}{|c|c|c|c|c|c|c|c|c|c|c|c|c|c|}
\hline 6 & 3 & 5.8960 & 4.8780 & 4.0560 & 3.1500 & 2.6290 & 1.9520 & 1.5430 & 1.1240 & 0.8970 & 0.6450 & 0.4970 & 0.3800 \\
\hline 6 & 3 & 5.4700 & 4.4450 & 3.3380 & 2.6570 & 2.0000 & 1.4880 & 1.1210 & 0.8180 & 0.6200 & 0.3740 & 0.1970 & 0.1600 \\
\hline 7 & 3 & 5.6710 & 4.9040 & 4.0310 & 3.1720 & 2.6380 & 2.1140 & 1.5650 & 1.1790 & 0.8880 & 0.6620 & 0.4850 & 0.3720 \\
\hline 7 & 3 & 5.8960 & 4.8780 & 4.0560 & 3.1500 & 2.6290 & 1.9520 & 1.5430 & 1.1240 & 0.8970 & 0.6450 & 0.4970 & 0.3800 \\
\hline 7 & 3 & 5.4700 & 4.4450 & 3.3380 & 2.6570 & 2.0000 & 1.4880 & 1.1210 & 0.8180 & 0.6200 & 0.3740 & 0.1970 & 0.1600 \\
\hline 1 & 4 & 6.1990 & 5.0820 & 4.1830 & 3.2120 & 2.6060 & 2.0040 & 1.6190 & 1.1240 & 0.8470 & 0.6350 & 0.4650 & 0.3300 \\
\hline 1 & 4 & 5.6090 & 4.6200 & 3.4330 & 2.6820 & 1.9950 & 1.4860 & 1.0900 & 0.7500 & 0.5210 & 0.3040 & 0.1450 & 0.0700 \\
\hline 1 & 4 & 5.7850 & 4.7770 & 3.6540 & 2.8920 & 2.3250 & 1.7080 & 1.2790 & 0.9120 & 0.6920 & 0.4510 & 0.2740 & 0.1390 \\
\hline 2 & 4 & 6.6040 & 5.1660 & 4.1430 & 3.2360 & 2.7340 & 2.0370 & 1.6580 & 1.1740 & 0.8450 & 0.6430 & 0.4590 & 0.3050 \\
\hline 2 & 4 & 6.3650 & 5.1620 & 4.1390 & 3.3380 & 2.6770 & 2.0490 & 1.5620 & 1.1460 & 0.8560 & 0.6160 & 0.4580 & 0.3070 \\
\hline 2 & 4 & 6.2810 & 5.7980 & 4.8260 & 3.6300 & 3.2270 & 2.5930 & 2.0910 & 1.5620 & 1.1740 & 0.8750 & 0.6640 & 0.4900 \\
\hline 3 & 4 & 5.6400 & 4.9500 & 4.1280 & 3.5080 & 2.8930 & 2.4160 & 1.9650 & 1.5800 & 1.2200 & 0.9630 & 0.7190 & 0.5670 \\
\hline 3 & 4 & 6.2510 & 5.2530 & 4.3410 & 3.5310 & 2.7820 & 2.2600 & 1.7530 & 1.2610 & 0.9250 & 0.7260 & 0.5520 & 0.3450 \\
\hline 3 & 4 & 5.9970 & 5.6060 & 4.4880 & 3.6970 & 3.1260 & 2.3290 & 1.8920 & 1.4380 & 1.1310 & 0.8530 & 0.6710 & 0.4480 \\
\hline 4 & 4 & 6.1380 & 5.0380 & 3.7140 & 2.9520 & 2.1920 & 1.6910 & 1.2260 & 0.8590 & 0.5550 & 0.2910 & 0.1820 & 0.0870 \\
\hline 4 & 4 & 6.1040 & 5.1950 & 3.8540 & 3.0560 & 2.2640 & 1.6790 & 1.2310 & 0.8400 & 0.5860 & 0.3300 & 0.1770 & 0.0870 \\
\hline 4 & 4 & 6.6290 & 5.2580 & 4.2700 & 3.3500 & 2.4630 & 1.8140 & 1.4230 & 0.9170 & 0.6950 & 0.3970 & 0.2370 & 0.1470 \\
\hline 5 & 4 & 5.7350 & 5.0250 & 4.1830 & 3.2920 & 2.6960 & 2.1230 & 1.6560 & 1.2690 & 0.9510 & 0.7520 & 0.5550 & 0.3860 \\
\hline 5 & 4 & 6.0260 & 5.4620 & 4.2110 & 3.6410 & 2.8020 & 2.1950 & 1.6970 & 1.2610 & 0.9940 & 0.7440 & 0.5430 & 0.3820 \\
\hline 5 & 4 & 6.1970 & 5.9370 & 4.6200 & 3.6530 & 3.0020 & 2.3550 & 1.7430 & 1.3750 & 1.0150 & 0.7470 & 0.5700 & 0.3290 \\
\hline 6 & 4 & 6.3150 & 5.0380 & 4.2200 & 3.2070 & 2.5860 & 1.9640 & 1.5780 & 1.1400 & 0.8070 & 0.6250 & 0.4580 & 0.2880 \\
\hline 6 & 4 & 6.2290 & 5.3550 & 4.4570 & 3.5970 & 2.8970 & 2.3110 & 1.7500 & 1.3090 & 1.0040 & 0.8020 & 0.5390 & 0.3590 \\
\hline 6 & 4 & 6.7950 & 5.1800 & 4.2580 & 3.1860 & 2.4690 & 1.8530 & 1.3990 & 0.9740 & 0.6950 & 0.4190 & 0.2250 & 0.1460 \\
\hline 7 & 4 & 6.3150 & 5.0380 & 4.2200 & 3.2070 & 2.5860 & 1.9640 & 1.5780 & 1.1400 & 0.8070 & 0.6250 & 0.4580 & 0.2880 \\
\hline 7 & 4 & 6.2290 & 5.3550 & 4.4570 & 3.5970 & 2.8970 & 2.3110 & 1.7500 & 1.3090 & 1.0040 & 0.8020 & 0.5390 & 0.3590 \\
\hline 7 & 4 & 6.7950 & 5.1800 & 4.2580 & 3.1860 & 2.4690 & 1.8530 & 1.3990 & 0.9740 & 0.6950 & 0.4190 & 0.2250 & 0.1460 \\
\hline
\end{tabular}


Table S8. Example of matrix input of variables for building the PCA model (Part 5).

\begin{tabular}{|c|c|c|c|c|c|c|c|c|c|c|c|c|}
\hline Day & $\begin{array}{c}\text { Crude } \\
\text { oil }\end{array}$ & $\begin{array}{c}\mathrm{DBE} \\
\mathrm{N}[\mathrm{H}] \\
25\end{array}$ & $\begin{array}{c}\mathrm{DBE} \\
\mathrm{N}[\mathrm{H}] \\
26\end{array}$ & $\begin{array}{c}\mathrm{DBE} \\
\mathrm{N}[\mathrm{H}] \\
27\end{array}$ & $\begin{array}{c}\mathrm{DBE} \\
\mathrm{N}[\mathrm{H}] \\
28\end{array}$ & $\begin{array}{c}\mathrm{DBE} \\
\mathrm{N}[\mathrm{H}] \\
29\end{array}$ & $\begin{array}{c}\mathrm{DBE} \\
\mathrm{N}[\mathrm{H}] \\
30\end{array}$ & $\begin{array}{c}\mathrm{DBE} \\
\mathrm{N}[\mathrm{H}] \\
31\end{array}$ & $\begin{array}{c}\mathrm{DBE} \\
\mathrm{N}[\mathrm{H}] \\
33\end{array}$ & $\begin{array}{c}\mathrm{DBE} \\
\mathrm{N}[\mathrm{H}] \\
37\end{array}$ & $\begin{array}{c}\mathrm{DBE} \\
\mathrm{N}[\mathrm{H}] \\
38\end{array}$ & $\begin{array}{c}\mathrm{DBE} \\
\mathrm{N}[\mathrm{H}] \\
39\end{array}$ \\
\hline 1 & 1 & 0.4540 & 0.2380 & 0.1230 & 0.0000 & 0.0000 & 0.0000 & 0.0000 & 0.0000 & 0.0000 & 0.0000 & 0.0000 \\
\hline 1 & 1 & .7090 & 0.5760 & 0.4420 & 0.3080 & 0.2520 & 0.1460 & 0.0770 & 0.0000 & 0.0000 & 0.0000 & 0.0210 \\
\hline 1 & 1 & 0.7250 & 0.5400 & 0.4040 & 0.3250 & 0.2390 & 0.1520 & 0.0560 & 0.0000 & 0.0000 & 0.0000 & 0.0000 \\
\hline 2 & 1 & 0.7370 & 0.5920 & 0.4890 & 0.3070 & 0.1790 & 0.0940 & 0.0000 & 0.0000 & 0.0000 & 0.0000 & 0.0000 \\
\hline 2 & 1 & 0.7450 & 0.5920 & 0.4550 & 0.2440 & 0.1620 & 0.0800 & 0.0000 & 0.0000 & 0.0000 & 0.0000 & 0.0000 \\
\hline 2 & 1 & 0.8510 & 0.6090 & 0.5200 & 0.4060 & 0.2690 & 0.1960 & 0.0940 & 0.0000 & 0.0000 & 0.0000 & 0.0210 \\
\hline 3 & 1 & 0.7390 & 0.6110 & 0.4780 & 0.3340 & 0.2560 & 0.1590 & 0.0660 & 0.0000 & 0.0000 & 0.0000 & 0.0000 \\
\hline 3 & 1 & 0.7470 & 0.6220 & 0.4650 & 0.3620 & 0.2590 & 0.1640 & 0.0700 & 0.0000 & 0.0000 & 0.0000 & 0.0200 \\
\hline 3 & 1 & 0.6790 & 0.5780 & 0.4710 & 0.3450 & 0.2430 & 0.1400 & 0.0600 & 0.0000 & 0.0000 & 0.0000 & 0.0170 \\
\hline 4 & 1 & & & & & & & & & & 0.0000 & 0.0000 \\
\hline 4 & 1 & 0.3330 & 0.1710 & 0.0970 & 0.0680 & 0.0000 & 0.0000 & 0.0000 & 0.0000 & 0.0000 & 0.0000 & 0.0000 \\
\hline 4 & 1 & 0.3860 & 0.2290 & 0.1010 & 0.0330 & 0.0000 & 0.0000 & 0.0000 & 0.0000 & 0.0000 & 0.0000 & 0.0000 \\
\hline 5 & 1 & & & & & & & & & & 0.0000 & 0.0000 \\
\hline 5 & 1 & 0.5740 & 0.4120 & 0.3120 & 0.1770 & 0.1080 & 0.0660 & 0.0000 & 0.0000 & 0.0000 & 0.0000 & 0.0000 \\
\hline 5 & 1 & 0.6830 & 0.5850 & 0.4580 & 0.3470 & 0.2110 & 0.0900 & 0.0250 & 0.0000 & 0.0000 & 0.0000 & 0.0000 \\
\hline 6 & 1 & 0.4600 & 0.2950 & 0.1510 & 0.0720 & 0.0200 & 0.0000 & 0.0000 & 0.0000 & 0.0000 & 0.0000 & 0.0000 \\
\hline 6 & 1 & 0.2280 & 0.0890 & 0.0180 & 0.0000 & 0.0000 & 0.0000 & 0.0000 & 0.0000 & 0.0000 & 0.0000 & 0.0000 \\
\hline 6 & 1 & 0.6370 & 0.5420 & 0.4500 & 0.3110 & 0.2230 & 0.1470 & 0.0610 & 0.0000 & 0.0000 & 0.0000 & 0.0130 \\
\hline 7 & 1 & 0.4600 & 0.2950 & 0.1510 & 0.0720 & 0.0200 & 0.0000 & 0.0000 & 0.0000 & 0.0000 & 0.0000 & 0.0000 \\
\hline 7 & 1 & 0.2280 & 0.0890 & 0.0180 & 0.0000 & 0.0000 & 0.0000 & 0.0000 & 0.0000 & 0.0000 & 0.0000 & 0.0000 \\
\hline 7 & 1 & 0.6370 & 0.5420 & 0.4500 & 0.3110 & 0.2230 & 0.1470 & 0.0610 & 0.0000 & 0.0000 & 0.0000 & 0.0130 \\
\hline 1 & 2 & 0.5510 & 0.4370 & 0.2750 & 0.0000 & 0.2260 & 0.0000 & 0.0000 & 0.1510 & 0.0520 & 0.0000 & 0.0000 \\
\hline 1 & 2 & 0.2320 & 0.1860 & 0.0950 & 0.0000 & 0.0230 & 0.0000 & 0.0000 & 0.0000 & 0.0000 & 0.0000 & 0.0000 \\
\hline 1 & 2 & 0.4180 & 0.2920 & 0.1580 & 0.0000 & 0.0520 & 0.0000 & 0.0000 & 0.0000 & 0.0000 & 0.0000 & 0.0000 \\
\hline 2 & 2 & 0.5370 & 0.3640 & 0.2600 & 0.0000 & 0.1610 & 0.0000 & 0.0000 & 0.0630 & 0.0000 & 0.0000 & 0.0000 \\
\hline 2 & 2 & 0.5190 & 0.3510 & 0.2470 & 0.0000 & 0.1500 & 0.0000 & 0.0000 & 0.0670 & 0.0000 & 0.0000 & 0.0000 \\
\hline
\end{tabular}




\begin{tabular}{|c|c|c|c|c|c|c|c|c|c|c|c|c|}
\hline 2 & 2 & 0.3350 & 0.1910 & 0.0870 & 0.0000 & 0.0180 & 0.0000 & 0.0000 & 0.0000 & 0.0000 & 0.0000 & 0.0000 \\
\hline 3 & 2 & 0.7210 & 0.4840 & 0.3810 & 0.0000 & 0.3030 & 0.0000 & 0.0000 & 0.2350 & 0.1590 & 0.0830 & 0.0000 \\
\hline 3 & 2 & 0.6970 & 0.4460 & 0.3530 & 0.0000 & 0.2860 & 0.0000 & 0.0000 & 0.2290 & 0.1470 & 0.0670 & 0.0000 \\
\hline 3 & 2 & 0.7370 & 0.4440 & 0.3770 & 0.0000 & 0.2700 & 0.0000 & 0.0000 & 0.2340 & 0.1650 & 0.0730 & 0.0000 \\
\hline 4 & 2 & 0.1190 & 0.0310 & 0.0000 & 0.0000 & 0.0000 & 0.0000 & 0.0000 & 0.0000 & 0.0000 & 0.0000 & 0.0000 \\
\hline 4 & 2 & 0.1470 & 0.0600 & 0.0000 & 0.0000 & 0.0000 & 0.0000 & 0.0000 & 0.0000 & 0.0000 & 0.0000 & 0.0000 \\
\hline 4 & 2 & 0.1560 & 0.0640 & 0.0000 & 0.0000 & 0.0000 & 0.0000 & 0.0000 & 0.0000 & 0.0000 & 0.0000 & 0.0000 \\
\hline 5 & 2 & 0.2220 & 0.1030 & 0.0290 & 0.0000 & 0.0000 & 0.0000 & 0.0000 & 0.0000 & 0.0000 & 0.0000 & 0.0000 \\
\hline 5 & 2 & 0.3650 & 0.2560 & 0.1610 & 0.0000 & 0.0540 & 0.0000 & 0.0000 & 0.0000 & 0.0000 & 0.0000 & 0.0000 \\
\hline 5 & 2 & 0.2530 & 0.1320 & 0.0000 & 0.0000 & 0.0000 & 0.0000 & 0.0000 & 0.0000 & 0.0000 & 0.0000 & 0.0000 \\
\hline 6 & 2 & 0.5280 & 0.3360 & 0.2440 & 0.0000 & 0.1530 & 0.0000 & 0.0000 & 0.0750 & 0.0000 & 0.0000 & 0.0000 \\
\hline 6 & 2 & 0.5370 & 0.3640 & 0.2600 & 0.0000 & 0.1610 & 0.0000 & 0.0000 & 0.0630 & 0.0000 & 0.0000 & 0.0000 \\
\hline 6 & 2 & 0.5030 & 0.3720 & 0.2620 & 0.0000 & 0.1460 & 0.0000 & 0.0000 & 0.0620 & 0.0000 & 0.0000 & 0.0000 \\
\hline 7 & 2 & 0.5280 & 0.3360 & 0.2440 & 0.0000 & 0.1530 & 0.0000 & 0.0000 & 0.0750 & 0.0000 & 0.0000 & 0.0000 \\
\hline 7 & 2 & 0.5370 & 0.3640 & 0.2600 & 0.0000 & 0.1610 & 0.0000 & 0.0000 & 0.0630 & 0.0000 & 0.0000 & 0.0000 \\
\hline 7 & 2 & 0.5020 & 0.3570 & 0.2620 & 0.0000 & 0.1460 & 0.0000 & 0.0000 & 0.0620 & 0.0000 & 0.0000 & 0.0000 \\
\hline 1 & 3 & 0.1580 & 0.0480 & 0.0000 & 0.0000 & 0.0000 & 0.0000 & 0.0000 & 0.0000 & 0.0000 & 0.0000 & 0.0000 \\
\hline 1 & 3 & 0.2290 & 0.1220 & 0.0410 & 0.0000 & 0.0000 & 0.0000 & 0.0000 & 0.0000 & 0.0000 & 0.0000 & 0.0000 \\
\hline 1 & 3 & 0.2710 & 0.1560 & 0.0540 & 0.0000 & 0.0000 & 0.0000 & 0.0000 & 0.0000 & 0.0000 & 0.0000 & 0.0000 \\
\hline 2 & 3 & 0.1090 & 0.0240 & 0.0000 & 0.0000 & 0.0000 & 0.0000 & 0.0000 & 0.0000 & 0.0000 & 0.0000 & 0.0000 \\
\hline 2 & 3 & 0.1580 & 0.0500 & 0.0000 & 0.0000 & 0.0000 & 0.0000 & 0.0000 & 0.0000 & 0.0000 & 0.0000 & 0.0000 \\
\hline 2 & 3 & 0.3430 & 0.2520 & 0.1850 & 0.0890 & 0.0170 & 0.0000 & 0.0000 & 0.0000 & 0.0000 & 0.0000 & 0.0000 \\
\hline 3 & 3 & 0.2620 & 0.1460 & 0.0620 & 0.0000 & 0.0000 & 0.0000 & 0.0000 & 0.0000 & 0.0000 & 0.0000 & 0.0000 \\
\hline 3 & 3 & 0.3560 & 0.2750 & 0.1790 & 0.0890 & 0.0180 & 0.0000 & 0.0000 & 0.0000 & 0.0000 & 0.0000 & 0.0000 \\
\hline 3 & 3 & 0.3230 & 0.2420 & 0.1620 & 0.0590 & 0.0000 & 0.0000 & 0.0000 & 0.0000 & 0.0000 & 0.0000 & 0.0000 \\
\hline 4 & 3 & 0.0770 & 0.0000 & 0.0000 & 0.0000 & 0.0000 & 0.0000 & 0.0000 & 0.0000 & 0.0000 & 0.0000 & 0.0000 \\
\hline 4 & 3 & 0.0430 & 0.0000 & 0.0000 & 0.0000 & 0.0000 & 0.0000 & 0.0000 & 0.0000 & 0.0000 & 0.0000 & 0.0000 \\
\hline 4 & 3 & 0.0460 & 0.0000 & 0.0000 & 0.0000 & 0.0000 & 0.0000 & 0.0000 & 0.0000 & 0.0000 & 0.0000 & 0.0000 \\
\hline 5 & 3 & 0.1520 & 0.0690 & 0.0000 & 0.0000 & 0.0000 & 0.0000 & 0.0000 & 0.0000 & 0.0000 & 0.0000 & 0.0000 \\
\hline 5 & 3 & 0.2280 & 0.0890 & 0.0180 & 0.0000 & 0.0000 & 0.0000 & 0.0000 & 0.0000 & 0.0000 & 0.0000 & 0.0000 \\
\hline 5 & 3 & 0.1740 & 0.0860 & 0.0190 & 0.0000 & 0.0000 & 0.0000 & 0.0000 & 0.0000 & 0.0000 & 0.0000 & 0.0000 \\
\hline 6 & 3 & 0.2290 & 0.0990 & 0.0240 & 0.0000 & 0.0000 & 0.0000 & 0.0000 & 0.0000 & 0.0000 & 0.0000 & 0.0000 \\
\hline
\end{tabular}




\begin{tabular}{|c|c|c|c|c|c|c|c|c|c|c|c|c|}
\hline 6 & 3 & 0.2340 & 0.1360 & 0.0460 & 0.0000 & 0.0000 & 0.0000 & 0.0000 & 0.0000 & 0.0000 & 0.0000 & 0.0000 \\
\hline 6 & 3 & 0.0510 & 0.0000 & 0.0000 & 0.0000 & 0.0000 & 0.0000 & 0.0000 & 0.0000 & 0.0000 & 0.0000 & 0.0000 \\
\hline 7 & 3 & 0.2290 & 0.0990 & 0.0240 & 0.0720 & 0.0200 & 0.0000 & 0.0000 & 0.0000 & 0.0000 & 0.0000 & 0.0000 \\
\hline 7 & 3 & 0.2340 & 0.1360 & 0.0460 & 0.0000 & 0.0000 & 0.0000 & 0.0000 & 0.0000 & 0.0000 & 0.0000 & 0.0000 \\
\hline 7 & 3 & 0.0510 & 0.0000 & 0.0000 & 0.0000 & 0.0000 & 0.0000 & 0.0000 & 0.0000 & 0.0000 & 0.0000 & 0.0000 \\
\hline 1 & 4 & 0.1440 & 0.0740 & 0.0000 & 0.0000 & 0.0000 & 0.0000 & 0.0000 & 0.0000 & 0.0000 & 0.0000 & 0.0000 \\
\hline 1 & 4 & 0.0000 & 0.0000 & 0.0000 & 0.0000 & 0.0000 & 0.0000 & 0.0000 & 0.0000 & 0.0000 & 0.0000 & 0.0000 \\
\hline 1 & 4 & 0.0640 & 0.0000 & 0.0000 & 0.0000 & 0.0000 & 0.0000 & 0.0000 & 0.0000 & 0.0000 & 0.0000 & 0.0000 \\
\hline 2 & 4 & 0.1360 & 0.0640 & 0.0000 & 0.0000 & 0.0000 & 0.0000 & 0.0000 & 0.0000 & 0.0000 & 0.0000 & 0.0000 \\
\hline 2 & 4 & 0.1300 & 0.0790 & 0.0140 & 0.0000 & 0.0000 & 0.0000 & 0.0000 & 0.0000 & 0.0000 & 0.0000 & 0.0000 \\
\hline 2 & 4 & 0.2930 & 0.1400 & 0.0600 & 0.0000 & 0.0000 & 0.0000 & 0.0000 & 0.0000 & 0.0000 & 0.0000 & 0.0000 \\
\hline 3 & 4 & 0.3530 & 0.1810 & 0.1040 & 0.0000 & 0.0000 & 0.0000 & 0.0000 & 0.0000 & 0.0000 & 0.0000 & 0.0000 \\
\hline 3 & 4 & 0.1760 & 0.0900 & 0.0450 & 0.0000 & 0.0000 & 0.0000 & 0.0000 & 0.0000 & 0.0000 & 0.0000 & 0.0000 \\
\hline 3 & 4 & 0.2950 & 0.1580 & 0.0900 & 0.0310 & 0.0000 & 0.0000 & 0.0000 & 0.0000 & 0.0000 & 0.0000 & 0.0000 \\
\hline 4 & 4 & 0.0000 & 0.0000 & 0.0000 & 0.0000 & 0.0000 & 0.0000 & 0.0000 & 0.0000 & 0.0000 & 0.0000 & 0.0000 \\
\hline 4 & 4 & 0.0000 & 0.0000 & 0.0000 & 0.0000 & 0.0000 & 0.0000 & 0.0000 & 0.0000 & 0.0000 & 0.0000 & 0.0000 \\
\hline 4 & 4 & 0.0300 & 0.0000 & 0.0000 & 0.0000 & 0.0000 & 0.0000 & 0.0000 & 0.0000 & 0.0000 & 0.0000 & 0.0000 \\
\hline 5 & 4 & 0.1900 & 0.1180 & 0.0540 & 0.0000 & 0.0000 & 0.0000 & 0.0000 & 0.0000 & 0.0000 & 0.0000 & 0.0000 \\
\hline 5 & 4 & 0.1810 & 0.0970 & 0.0480 & 0.0000 & 0.0000 & 0.0000 & 0.0000 & 0.0000 & 0.0000 & 0.0000 & 0.0000 \\
\hline 5 & 4 & 0.1830 & 0.0870 & 0.0000 & 0.0000 & 0.0000 & 0.0000 & 0.0000 & 0.0000 & 0.0000 & 0.0000 & 0.0000 \\
\hline 6 & 4 & 0.1310 & 0.0590 & 0.0000 & 0.0000 & 0.0000 & 0.0000 & 0.0000 & 0.0000 & 0.0000 & 0.0000 & 0.0000 \\
\hline 6 & 4 & 0.1660 & 0.0940 & 0.0340 & 0.0000 & 0.0000 & 0.0000 & 0.0000 & 0.0000 & 0.0000 & 0.0000 & 0.0000 \\
\hline 6 & 4 & 0.0360 & 0.0000 & 0.0000 & 0.0000 & 0.0000 & 0.0000 & 0.0000 & 0.0000 & 0.0000 & 0.0000 & 0.0000 \\
\hline 7 & 4 & 0.1310 & 0.0590 & 0.0000 & 0.0000 & 0.0000 & 0.0000 & 0.0000 & 0.0000 & 0.0000 & 0.0000 & 0.0000 \\
\hline 7 & 4 & 0.1660 & 0.0940 & 0.0340 & 0.0000 & 0.0000 & 0.0000 & 0.0000 & 0.0000 & 0.0000 & 0.0000 & 0.0000 \\
\hline 7 & 4 & 0.0360 & 0.0000 & 0.0000 & 0.0000 & 0.0000 & 0.0000 & 0.0000 & 0.0000 & 0.0000 & 0.0000 & 0.0000 \\
\hline
\end{tabular}


Table S9. Intermediate precision limit of properties of ESI(+)FT-ICR MS and ESI(+)Orbitrap MS properties for Crude oils A, B, C, and D.

\begin{tabular}{|c|c|c|c|c|c|c|c|c|}
\hline $\begin{array}{c}\text { Mass } \\
\text { Spectrometer }\end{array}$ & & $\mathrm{ESI}(+) \mathrm{F}^{-}$ & ICR MS & & & $\mathrm{ESI}(+) \mathrm{Or}$ & itrap MS & \\
\hline Crude oil & A & B & C & D & A & B & C & D \\
\hline$\%$ Procedure & 16,2080 & 46,3465 & 11,9993 & 12,8777 & 5,9106 & 1,7972 & 3,2639 & 3,7802 \\
\hline $\begin{array}{c}\text { Total } \\
\text { compounds }\end{array}$ & 913,6683 & $\begin{array}{c}711,619 \\
2 \\
\end{array}$ & $\begin{array}{c}839,649 \\
2 \\
\end{array}$ & $\begin{array}{c}734,714 \\
6 \\
\end{array}$ & $\begin{array}{c}1037,343 \\
4 \\
\end{array}$ & $\begin{array}{c}591,345 \\
1 \\
\end{array}$ & $\begin{array}{c}508,549 \\
8 \\
\end{array}$ & 702,5621 \\
\hline $\begin{array}{l}\text { Classes } \\
\text { Number }\end{array}$ & 4,2179 & 7,7932 & 4,9511 & 4,7435 & 3,6039 & 2,1170 & 2,3230 & 2,5303 \\
\hline $\begin{array}{c}\% \text { of } \mathrm{N}[\mathrm{H}] \\
\text { Class }\end{array}$ & 21,2849 & 41,0127 & 11,1935 & 13,3072 & 14,1431 & 2,8215 & 2,7274 & 11,6314 \\
\hline $\begin{array}{c}\text { Total } \mathrm{N}^{\circ} \text { of } \\
\mathrm{N}[\mathrm{H}] \\
\text { compounds }\end{array}$ & 196,0042 & $\begin{array}{c}229,105 \\
3\end{array}$ & $\begin{array}{c}204,216 \\
1\end{array}$ & $\begin{array}{c}235,167 \\
3\end{array}$ & 396,9783 & $\begin{array}{c}305,011 \\
2\end{array}$ & $\begin{array}{c}310,181 \\
3\end{array}$ & 197,5379 \\
\hline$\% \mathrm{NO}[\mathrm{H}]$ Class & 1,5625 & 1,8674 & 1,3322 & 1,7243 & 6,1443 & 2,9320 & 2,2196 & 6,7710 \\
\hline $\begin{array}{c}\text { Total } \mathrm{N}^{\circ} \text { of } \\
\mathrm{NO}[\mathrm{H}] \\
\text { compounds }\end{array}$ & 120,0420 & $\begin{array}{c}134,025 \\
3\end{array}$ & $\begin{array}{c}182,016 \\
4\end{array}$ & $\begin{array}{c}120,862 \\
6\end{array}$ & 451,5256 & $\begin{array}{c}242,686 \\
7\end{array}$ & $\begin{array}{c}240,677 \\
3\end{array}$ & $\begin{array}{c}1061,414 \\
3\end{array}$ \\
\hline$\% \mathrm{OS}[\mathrm{H}]$ Class & 0,1574 & 0,3285 & 0,8064 & 0,2991 & 7,5602 & 0,2527 & 0,2002 & 3,1946 \\
\hline $\begin{array}{c}\text { Total } \mathrm{N}^{\circ} \text { of } \\
\text { OS }[\mathrm{H}] \\
\text { compounds }\end{array}$ & 7,2836 & 40,6974 & 3,8966 & 10,5289 & 272,4653 & 72,5717 & 53,9435 & 194,9451 \\
\hline $\begin{array}{l}\text { \% N2S[H] } \\
\text { Class }\end{array}$ & 0,7103 & 0,7906 & 0,6372 & 0,3809 & 0,5496 & 1,0322 & 1,0818 & 0,5084 \\
\hline $\begin{array}{l}\text { Total } \mathrm{N}^{\circ} \text { of } \\
\mathrm{N} 2 \mathrm{~S}[\mathrm{H}] \\
\text { compounds }\end{array}$ & 58,5709 & 61,9792 & 32,0695 & 39,2676 & 89,3063 & $\begin{array}{c}125,090 \\
6\end{array}$ & $\begin{array}{c}150,419 \\
5\end{array}$ & 83,8240 \\
\hline$\% \mathrm{NS}[\mathrm{H}]$ Class & 2,6828 & 2,2331 & 2,2384 & 2,4798 & 0,7270 & 0,1645 & 0,1842 & - \\
\hline $\begin{array}{c}\text { Total } \mathrm{N}^{\circ} \text { of } \\
\mathrm{NS}[\mathrm{H}] \\
\text { compounds }\end{array}$ & 88,4827 & $\begin{array}{c}115,914 \\
9\end{array}$ & $\begin{array}{c}113,734 \\
7\end{array}$ & 80,3076 & 46,0168 & 11,8491 & 18,7119 & - \\
\hline $\begin{array}{c}\% \text { NOS}[\mathrm{H}] \\
\text { Class }\end{array}$ & 10,3754 & 3,7588 & 3,2144 & 4,5915 & & 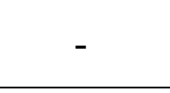 & - & - \\
\hline
\end{tabular}




\begin{tabular}{|c|c|c|c|c|c|c|c|c|}
\hline $\begin{array}{l}\text { Total } \mathrm{N}^{\circ} \text { of } \\
\text { NOS }[\mathrm{H}] \\
\text { compounds }\end{array}$ & 548,1119 & $\begin{array}{c}238,814 \\
2\end{array}$ & $\begin{array}{c}240,716 \\
6\end{array}$ & $\begin{array}{c}290,676 \\
9\end{array}$ & - & - & - & - \\
\hline$\% \mathrm{~N} 2[\mathrm{H}]$ Class & 1,9662 & 7,3845 & 3,9731 & 2,0599 & 1,3245 & 1,5056 & 1,7692 & 0,4974 \\
\hline $\begin{array}{c}\text { Total } \mathrm{N}^{\circ} \text { of } \\
\mathrm{N} 2[\mathrm{H}] \\
\text { compounds }\end{array}$ & 137,3413 & $\begin{array}{c}188,312 \\
6\end{array}$ & $\begin{array}{c}155,633 \\
1\end{array}$ & $\begin{array}{c}177,247 \\
3\end{array}$ & 173,1979 & $\begin{array}{c}157,578 \\
3\end{array}$ & $\begin{array}{c}129,380 \\
9\end{array}$ & 82,9823 \\
\hline $\begin{array}{l}\% \mathrm{~N} 2 \mathrm{O}[\mathrm{H}] \\
\text { Class }\end{array}$ & 0,1574 & 0,8005 & 0,0875 & 0,4877 & 0,3517 & - & 0,0271 & - \\
\hline $\begin{array}{l}\text { Total } \mathrm{N}^{\circ} \text { of } \\
\mathrm{N} 2 \mathrm{O}[\mathrm{H}] \\
\text { compounds }\end{array}$ & 7,8981 & $\begin{array}{c}111,981 \\
7\end{array}$ & 31,1375 & 45,6466 & 71,2315 & - & 3,3813 & - \\
\hline $\begin{array}{c}\% \mathrm{~N} 2 \mathrm{OS}[\mathrm{H}] \\
\text { Class }\end{array}$ & 0,0192 & 0,3703 & - & 0,1285 & - & 0,1076 & 0,1264 & - \\
\hline $\begin{array}{c}\text { Total } \mathrm{N}^{\circ} \text { of } \\
\mathrm{N} 2 \mathrm{OS}[\mathrm{H}] \\
\text { compounds }\end{array}$ & 7,0572 & 63,6630 & - & 4,4858 & - & 43,9738 & 53,4035 & - \\
\hline$\% \mathrm{O}[\mathrm{H}]$ Class & 0,2872 & 0,1134 & 0,1990 & 0,0908 & 0,0196 & - & - & 0,0296 \\
\hline $\begin{array}{l}\text { Total } \mathrm{N}^{\circ} \text { of } \\
\mathrm{O}[\mathrm{H}] \\
\text { compounds }\end{array}$ & 6,4865 & 40,2817 & 8,3375 & 2,5303 & 5,5930 & - & - & 4,4449 \\
\hline$\% \mathrm{HC}[\mathrm{H}]$ Class & 0,0908 & 0,1213 & 0,2235 & - & 0,0222 & - & - & 0,0272 \\
\hline $\begin{array}{c}\text { Total } \mathrm{N}^{\circ} \text { of } \\
\mathrm{HC}[\mathrm{H}] \\
\text { compounds }\end{array}$ & 2,5303 & 41,1844 & 7,5548 & - & 5,1857 & - & - & 4,5365 \\
\hline$\% \mathrm{~S}[\mathrm{H}]$ Class & 0,1819 & 0,6653 & 0,4550 & 0,0444 & - & - & 0,0296 & - \\
\hline $\begin{array}{c}\text { Total } \mathrm{N}^{\circ} \text { of } \\
\mathrm{S}[\mathrm{H}] \\
\text { compounds }\end{array}$ & 8,2048 & 81,7032 & 6,4441 & 3,7041 & - & - & 3,7041 & - \\
\hline DBE N[H] 3 & 0,6353 & 0,7985 & 0,8890 & 0,4807 & 0,2046 & 0,1125 & 0,1496 & 0,0845 \\
\hline DBE N[H] 4 & 1,6764 & 2,2627 & 2,4083 & 1,5253 & 1,0239 & 0,6152 & 0,7451 & 0,5560 \\
\hline $\mathrm{DBE} N[\mathrm{H}] 5$ & 2,7164 & 3,4772 & 3,5617 & 2,6498 & 1,9194 & 1,2228 & 1,5902 & 1,6473 \\
\hline $\mathrm{DBE} N[\mathrm{H}] 6$ & 3,5803 & 4,2418 & 4,1818 & 3,6166 & 2,7314 & 1,6358 & 2,6519 & 2,5175 \\
\hline DBE N[H] 7 & 3,9605 & 4,5527 & 4,5929 & 4,1130 & 3,2513 & 1,5342 & 2,0513 & 2,4536 \\
\hline $\mathrm{DBE} N[\mathrm{H}] 8$ & 4,2232 & 4,7421 & 4,6096 & 4,1957 & 3,0261 & 1,4225 & 2,4048 & 2,7537 \\
\hline
\end{tabular}




\begin{tabular}{ccccccccc}
\hline DBE N[H] 9 & 4,1977 & 4,2590 & 4,3277 & 4,3033 & 2,2359 & 1,5360 & 1,4890 & 2,1182 \\
\hline DBE N[H] 10 & 4,0873 & 3,9592 & 3,9975 & 4,0631 & 1,9612 & 0,7449 & 1,2202 & 1,8796 \\
\hline DBE N[H] 11 & 3,9229 & 3,6574 & 3,6035 & 3,8727 & 1,3589 & 1,0698 & 0,7182 & 1,5735 \\
\hline DBE N[H] 12 & 3,6346 & 3,4052 & 3,3063 & 3,6601 & 1,0301 & 0,3014 & 0,8762 & 0,9798 \\
\hline DBE N[H] 13 & 3,4147 & 3,0668 & 2,9991 & 3,4024 & 1,0844 & 0,5841 & 0,8386 & 0,9299 \\
\hline DBE N[H] 14 & 3,1075 & 2,8161 & 2,6539 & 3,1393 & 0,8971 & 0,5680 & 0,8626 & 0,9316 \\
\hline DBE N[H] 15 & 2,7987 & 2,5230 & 2,2742 & 2,7858 & 0,8152 & 0,6571 & 1,1238 & 0,9259 \\
\hline DBE N[H] 16 & 2,4928 & 2,2255 & 1,9898 & 2,5182 & 0,8473 & 0,6547 & 1,0463 & 0,7313 \\
\hline DBE N[H] 17 & 2,1705 & 1,8631 & 1,6860 & 2,2738 & 0,7119 & 0,6775 & 1,1146 & 0,8006 \\
\hline DBE N[H] 18 & 1,9635 & 1,6699 & 1,4308 & 1,9847 & 0,8118 & 0,6300 & 0,9689 & 0,7604 \\
\hline DBE N[H] 19 & 1,6598 & 1,3701 & 1,2202 & 1,7562 & 0,8988 & 0,6164 & 0,8540 & 0,6823 \\
\hline DBE N[H] 20 & 1,4665 & 1,1825 & 0,9393 & 1,5443 & 0,9212 & 0,5207 & 0,6550 & 0,5808 \\
\hline DBE N[H] 21 & 1,2232 & 0,9442 & 0,9209 & 1,3098 & 0,8314 & 0,4609 & 0,5434 & 0,5092 \\
\hline DBE N[H] 22 & 1,0136 & 0,8035 & 0,5912 & 1,0585 & 0,7031 & 0,4690 & 0,5001 & 0,5001 \\
\hline DBE N[H] 23 & 0,8268 & 0,6187 & 0,5806 & 0,9666 & 0,6020 & 0,3376 & 0,4469 & 0,4282 \\
\hline DBE N[H] 24 & 0,7864 & 1,2163 & 0,8255 & 0,7335 & 0,5030 & 0,3208 & 0,3466 & 0,3442 \\
\hline DBE N[H] 25 & 0,6368 & 0,8177 & 0,3155 & 0,5851 & 0,4447 & 0,2730 & 0,2802 & 0,2450 \\
\hline DBE N[H] 26 & 0,5090 & 0,4642 & - & 0,4076 & 0,4966 & 0,2326 & 0,2409 & 0,1358 \\
\hline DBE N[H] 27 & 0,2660 & 0,3899 & - & 0,2023 & 0,4726 & 0,2045 & 0,1728 & 0,0843 \\
\hline DBE N[H] 28 & - & 0,2507 & - & - & 0,4146 & - & 0,096 & 0,021 \\
\hline DBE N[H] 29 & - & - & - & - & 0,3113 & 0,1798 & 0,0235 & - \\
\hline DBE N[H] 30 & - & - & - & - & 0,2173 & - & - & - \\
\hline DBE N[H] 31 & - & - & - & - & 0,1194 & - & - & - \\
\hline DBE N[H] 33 & - & - & - & - & - & 0,1263 & - & - \\
\hline DBE N[H] 37 & - & - & - & - & - & 0,0627 & - & - \\
\hline DBE N[H] 38 & - & - & - & - & - & 0,0260 & - & - \\
\hline DBE N[H] 39 & - & - & - & - & 0,0279 & - & - & - \\
\hline
\end{tabular}


Table S10. Intermediate precision limit of the ESI(-)FT-ICR MS and ESI(-)Orbitrap MS properties for Crude oils A, B, C, and D.

\begin{tabular}{|c|c|c|c|c|c|c|c|c|}
\hline \multirow{2}{*}{$\begin{array}{c}\text { Mass } \\
\text { Spectrometer } \\
\text { Crude Oil }\end{array}$} & \multicolumn{4}{|c|}{ ESI(-)FT-ICR MS } & \multicolumn{4}{|c|}{ ESI(-)Orbitrap MS } \\
\hline & $A$ & $B$ & $C$ & $D$ & $A$ & $\mathrm{~B}$ & $\mathrm{C}$ & $D$ \\
\hline$\%$ Procedure & 30,3333 & 24,1509 & 18,6155 & 25,1193 & 12,2953 & 23,1965 & 9,4847 & 15,1984 \\
\hline $\begin{array}{c}\text { Total } \\
\text { compounds }\end{array}$ & 898,8008 & 820,3762 & 1075,2043 & 1601,5292 & 1317,1280 & 440,6844 & 950,2122 & 681,5686 \\
\hline$\%$ of $\mathrm{N}[\mathrm{H}]$ Class & 16,1809 & 20,9313 & 17,8510 & 18,5129 & 15,3983 & 25,6387 & 19,2665 & 21,0911 \\
\hline $\begin{array}{l}\text { Total } \mathrm{N}^{\circ} \text { of } \mathrm{N}[\mathrm{H}] \\
\text { compounds }\end{array}$ & 165,1766 & 62,5323 & 109,7525 & 165,1354 & 256,9731 & 198,6878 & 206,4313 & 168,7761 \\
\hline$\% \mathrm{NO}[\mathrm{H}]$ Class & 2,4724 & 3,5762 & 4,7013 & 4,6790 & 5,2693 & 1,1511 & 4,7301 & 4,5172 \\
\hline $\begin{array}{c}\text { Total } \mathrm{N}^{\circ} \text { of } \\
\mathrm{NO}[\mathrm{H}] \\
\text { compounds }\end{array}$ & 215,1915 & 200,5442 & 197,5722 & 269,6697 & 312,2475 & 68,4037 & 211,6431 & 114,2246 \\
\hline$\% \mathrm{O}[\mathrm{H}]$ Class & 10,4435 & 3,7277 & 2,1277 & 9,1657 & 6,8282 & 5,7055 & 4,5143 & 7,4796 \\
\hline $\begin{array}{l}\text { Total } \mathrm{N}^{\circ} \text { of } \mathrm{O}[\mathrm{H}] \\
\text { compounds }\end{array}$ & 145,5311 & 82,3250 & 99,7470 & 191,5879 & 256,7270 & 96,3338 & 145,0450 & 195,1273 \\
\hline$\% \mathrm{O} 2[\mathrm{H}]$ Class & 4,7710 & 2,0412 & 4,8000 & 5,5996 & 13,8598 & 9,7993 & 8,3790 & 6,5567 \\
\hline $\begin{array}{c}\text { Total } \mathrm{N}^{0} \text { of } \\
\mathrm{O} 2[\mathrm{H}] \\
\text { compounds }\end{array}$ & 263,3844 & 114,1197 & 144,3887 & 373,2750 & 453,9742 & 120,5030 & 185,9486 & 185,9473 \\
\hline$\% \mathrm{O} 3[\mathrm{H}]$ Class & 0,8684 & 0,0889 & 0,2418 & 0,3472 & 6,6710 & 0,8136 & 1,9619 & 0,7093 \\
\hline $\begin{array}{c}\text { Total } \mathrm{N}^{\circ} \text { of } \\
\mathrm{O} 3[\mathrm{H}] \\
\text { compounds }\end{array}$ & 7,9673 & 4,4449 & 19,0294 & 52,1154 & 16,8443 & 10,9338 & 63,5919 & 21,3811 \\
\hline$\%$ OS $[\mathrm{H}]$ Class & 0,2991 & - & - & - & 6,6526 & - & - & - \\
\hline $\begin{array}{c}\text { Total } \mathrm{N}^{\circ} \text { of } \\
\text { OS }[\mathrm{H}] \\
\text { compounds }\end{array}$ & 13,6733 & - & - & - & 6,7015 & - & - & - \\
\hline$\%$ O3S[H] Class & 3,7619 & 1,0367 & 1,3205 & 3,6707 & 7,8223 & 6,9363 & 3,5877 & 10,4784 \\
\hline $\begin{array}{c}\text { Total } \mathrm{N}^{\circ} \text { of } \\
\mathrm{O} 3 \mathrm{~S}[\mathrm{H}] \\
\text { compounds }\end{array}$ & 23,0902 & 16,7162 & 30,1238 & 14,2498 & 8,6973 & 8,3101 & 12,8632 & 7,7873 \\
\hline
\end{tabular}




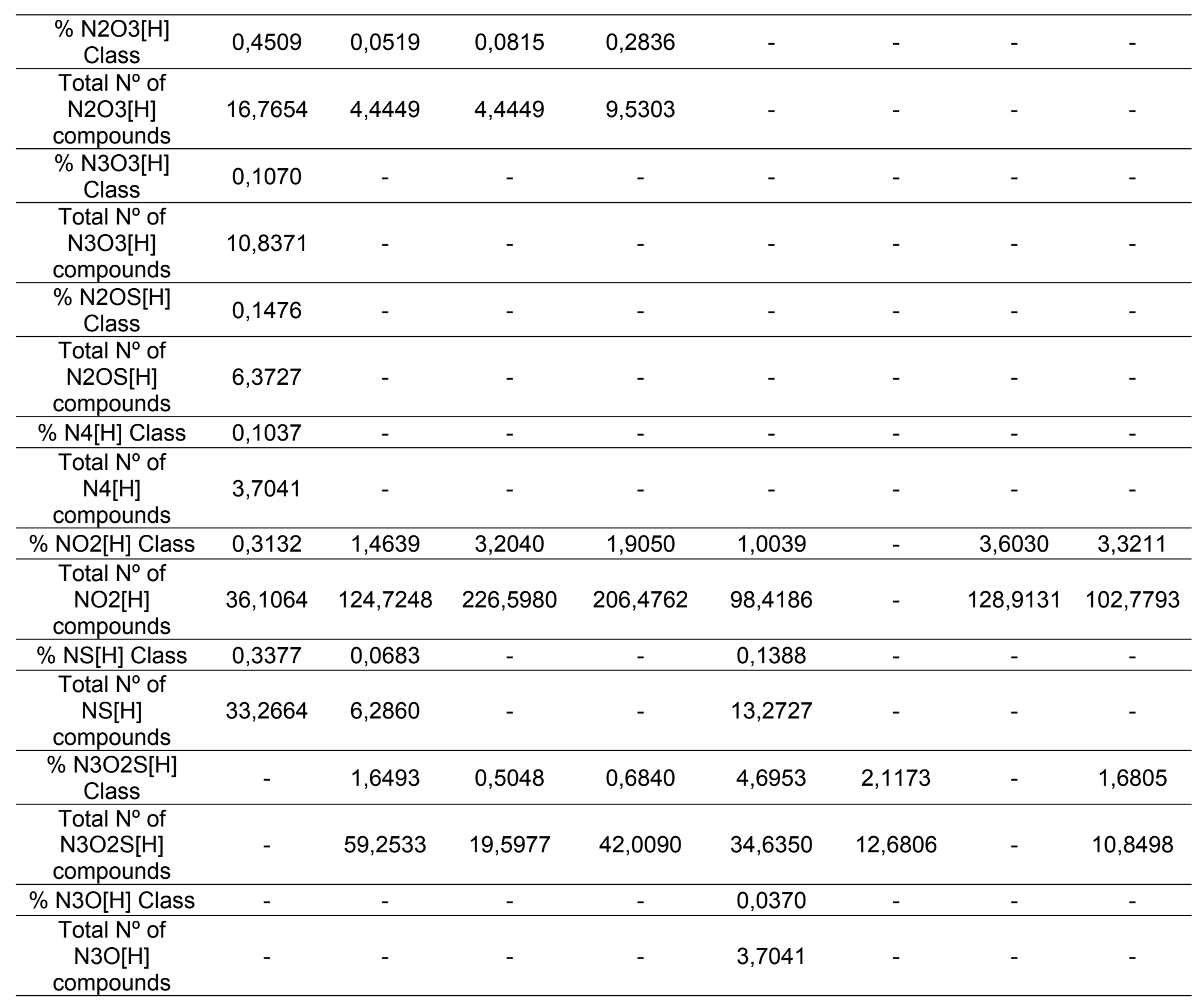




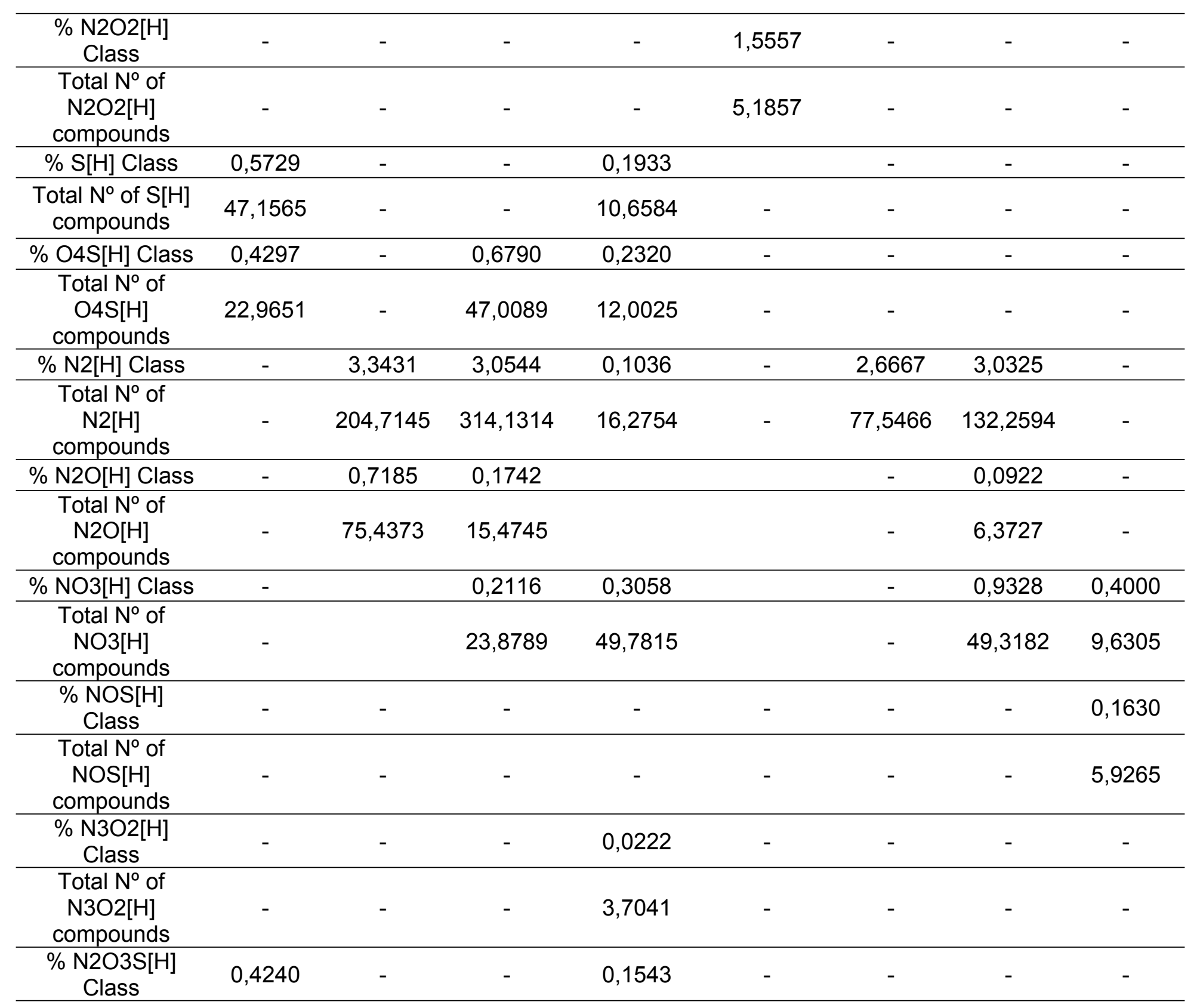




\begin{tabular}{ccccccccc}
\hline $\begin{array}{c}\text { Total No of } \\
\text { N2O3S[H] } \\
\text { compounds }\end{array}$ & 26,7667 & - & - & 19,8504 & - & - & - & - \\
\hline DBE N[H] 7 & & 0,1812 & 0,1523 & - & 0,0223 & 1,0817 & 0,5421 & - \\
\hline DBE N[H] 8 & 0,2521 & 0,1999 & 0,1182 & - & 0,2559 & 1,3129 & 0,5254 & - \\
\hline DBE N[H] 9 & 0,3502 & 0,2315 & 0,1349 & 0,0922 & 0,3162 & 1,0562 & 0,6911 & - \\
\hline DBE N[H] 10 & 0,7500 & 2,1183 & 2,4757 & 2,5603 & 2,9046 & 4,8691 & 4,0974 & 2,3772 \\
\hline DBE N[H] 11 & 0,5231 & 1,2538 & 2,0116 & 1,1936 & 2,1915 & 4,1703 & 2,8545 & 2,1705 \\
\hline DBE N[H] 12 & 0,3405 & 0,7130 & 1,3380 & 0,6197 & 1,9711 & 2,9343 & 1,3324 & 1,2126 \\
\hline DBE N[H] 13 & 1,2018 & 4,9899 & 4,3681 & 6,8940 & 5,0214 & 17,1336 & 13,1702 & 9,3095 \\
\hline DBE N[H] 14 & 0,8083 & 2,0135 & 2,3943 & 2,9832 & 2,8115 & 5,8295 & 2,8557 & 1,7760 \\
\hline DBE N[H] 15 & 0,5886 & 0,8552 & 1,3830 & 1,8041 & 1,8896 & 3,3895 & 1,3783 & 1,3899 \\
\hline DBE N[H] 16 & 0,8383 & 1,6438 & 1,8007 & 1,4921 & 2,1260 & 9,4165 & 3,5656 & 1,3160 \\
\hline DBE N[H] 17 & 0,5069 & 0,5932 & 0,8256 & 0,7095 & 1,3394 & 2,9312 & 1,6297 & 1,0159 \\
\hline DBE N[H] 18 & 0,2710 & 0,4764 & 0,5839 & 0,5335 & 1,2572 & 2,6908 & 0,7716 & 1,0595 \\
\hline DBE N[H] 19 & 0,2423 & 0,4093 & 0,5085 & 0,4781 & 1,2426 & 1,8472 & 1,0911 & 1,0153 \\
\hline DBE N[H] 20 & 0,2465 & 0,3251 & 0,3631 & 0,4799 & 1,0270 & 1,9278 & 1,9141 & 1,5461 \\
\hline DBE N[H] 21 & 0,2850 & 0,2955 & 0,3131 & 0,4463 & 0,8927 & 1,6914 & 0,7175 & 1,2040 \\
\hline DBE N[H] 22 & 0,3533 & 0,3161 & 0,1570 & 0,3279 & 0,6906 & 1,1337 & 0,7292 & 0,9161 \\
\hline DBE N[H] 23 & 0,3643 & 0,2319 & 0,2892 & 0,4318 & 0,5717 & 0,5670 & 0,4393 & 0,1282 \\
\hline DBE N[H] 24 & 0,2173 & 0,1986 & 0,1800 & 0,2101 & 0,3970 & - & 0,0736 & - \\
\hline DBE N[H] 25 & 0,0914 & 0,1641 & - & 0,0776 & 0,1903 & - & - & - \\
\hline DBE N[H] 26 & - & 0,1021 & - & - & 0,0575 & - & - & - \\
\hline
\end{tabular}

